\title{
The Unified Theory of Acceptance and Use of Technology (UTAUT): A Literature Review
}

\author{
Michael D Williams \\ School of Management, Swansea University \\ Swansea, UK \\ Nripendra P Rana \\ School of Management, Swansea University \\ Swansea, UK \\ Yogesh K Dwivedi ${ }^{1}$ \\ School of Management \\ Room \#1, Haldane Building \\ Swansea University, Singleton Park \\ Swansea, SA2 8PP, Wales, UK. \\ Email: ykdwivedi@gmail.com
}

\begin{abstract}
Purpose - The purpose of this paper is to perform a systematic review of articles that have used the unified theory of acceptance and use of technology (UTAUT).

Design/methodology/approach: The results produced in this research are based on the literature analysis of 174 existing articles on the UTAUT model. This has been performed by collecting data including demographic details, methodological details, limitations, and significance of relationships between the constructs from the available articles based on the UTAUT.

Findings: The findings were categorised by dividing the articles that used the UTAUT model into types of information systems used, research approach and methods employed, and tools and techniques implemented to analyse results. We also perform the weight analysis of variables and found that performance expectancy and behavioural intention qualified for the best predictor category. The research also analysed and presented the limitations of existing studies.
\end{abstract}

Research limitations/implications: The search activities were centered on occurrences of keywords to avoid tracing a large number of publications where these keywords might have been used as casual words in the main text. However, we acknowledge that there may be a number of studies, which lack keywords in the title, but still focus upon UTAUT in some form.

Originality/value: This is the first research of its type, which has extensively examined the literature on the UTAUT and provided the researchers with the accumulative knowledge about the model.

Keywords: UTAUT, Systematic review, Weight analysis, External variables, Demographic details, Methodological analysis

Paper type Research paper

\footnotetext{
${ }^{1}$ Corresponding Author
} 


\section{Introduction}

The continuing quest to ensure user acceptance of technology is an ongoing management challenge (Schwarz and Chin, 2007), and one that has occupied IS/IT researchers to such an extent that technology adoption and diffusion research is now considered to be among the more mature areas of exploration (Venkatesh et al., 2003). This substantial level of activity has witnessed the use of a wide range of exploratory techniques examining many different systems and technologies in countless different contexts, to the extent that even the most cursory examination of the extant body of literature will reveal a variety of stakeholder perspectives, technologies and contexts, units of analysis, theories, and research methods (Williams et al., 2009). This situation has in turn led to an element of confusion among researchers, as they are often forced to pick and choose characteristics across a wide variety of often competing models and theories. In response to this confusion, and in order to harmonize the literature associated with acceptance of new technology, Venkatesh et al. (2003) developed a unified model that brings together alternative views on user and innovation acceptance - The Unified Theory of Acceptance and Use of Technology (UTAUT).

The UTAUT (Figure 1) suggests that four core constructs (performance expectancy, effort expectancy, social influence and facilitating conditions) are direct determinants of behavioural intention and ultimately behaviour, and that these constructs are in turn moderated by gender, age, experience, and voluntariness of use (Venkatesh et al., 2003). It is argued that by examining the presence of each of these constructs in a 'real world' environment, researchers and practitioners will be able to asses an individual's intention to use a specific system, thus allowing for the identification of the key influences on acceptance in any given context. The theory was developed through the review and integration of eight dominant theories and models, viz: the Theory of Reasoned Action (TRA), the Technology Acceptance Model (TAM), the Motivational Model (MM), the Theory of Planned Behaviour (TPB), a combined Theory of Planned Behaviour/Technology Acceptance Model (C-TPBTAM), the Model of PC Utilization (MPCU), Innovation Diffusion Theory (IDT), and Social Cognitive Theory (SCT). These contributing theories and models have all been widely and successfully utilised by a large number of previous studies of technology or innovation adoption and diffusion within a range of disciplines including information systems, marketing, social psychology, and management. In their original article, Venkatesh et al. (2003) presented results from a six-month study of four organisations, which revealed that the eight contributing models explained between 17 and 53 percent of variance in user intentions to use IT. However, UTAUT was found to outperform the eight individual models with an adjusted $\mathrm{R}^{2}$ of $69 \%$ (Venkatesh et al., 2003).

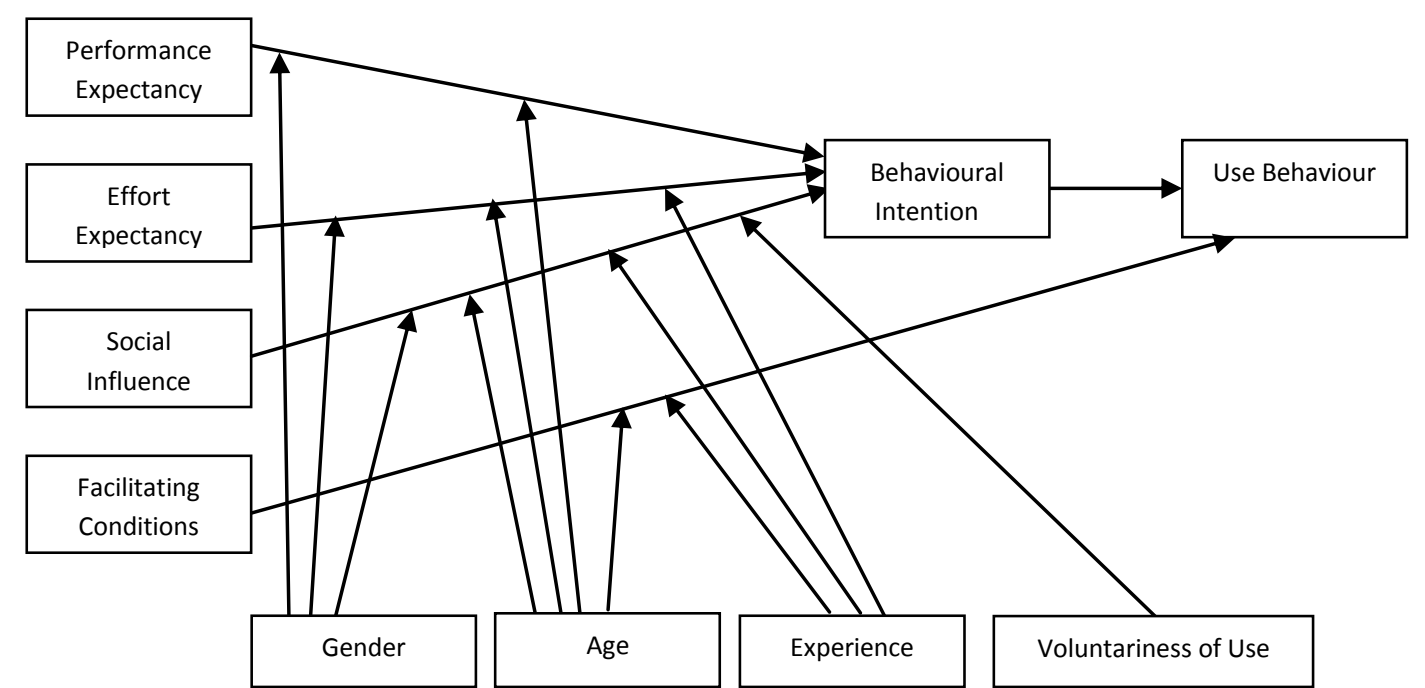


Fig. 1. Unified Theory of Acceptance and Use of Technology (Source: Venkatesh et al., 2003)

In the years since its introduction, UTAUT has been widely employed in technology adoption and diffusion research as a theoretical lens by researchers conducting empirical studies of user intention and behaviour. At the time of writing, the original article Venkatesh et al. (2003) has been cited just under 5000 times, with UTAUT being discussed with reference to a range of technologies (including the Internet, Web sites, Hospital Information Systems, Tax Payment Systems and Mobile Technology among others) with different control factors (such as age, gender, experience, voluntariness to use, income, and education), and focusing upon a variety of user groups (for instance, students, professionals, and general users).

However, despite this evident impact, no study to date has either surveyed or reviewed the performance of UTAUT, or explored/assessed the findings, limitations, and potential future directions. In keeping with other review work such as that of Lee et al. (2003), such a study is likely to be of value in that it can assist researchers of acceptance and use understand prior UTAUT-related findings, recognize possible future research topics, and guide future research endeavours. The aim of this study therefore is to provide such a review.

The remainder of this paper is structured as follows: In the following section we describe the methodology employed, and follow this with a section presenting our findings based upon an analysis of the material along a series of dimensions - including demographic aspects, types of technology examined, methodological considerations, and an analysis of UTAUT and external constructs employed in various studies. We present a summary of the limitations of extant UTAUT studies, and finally we present our concluding remarks and suggestions for future research directions.

\section{Research Methodology}

This study examines UTAUT research conducted from 2004 to June 2011. A comprehensive electronic search using ISI Web of Knowledge and Google Scholar resulted in 174 usable research papers. We used the keywords such as "unified theory of acceptance and use of technology" or "UTAUT" to perform the overall search in the title and/or abstract of the articles. The studies, although being scattered across 134 journals and conferences, appeared more frequently in journals such as Computers in Human Behaviour, Computers \& Education, Communications of the Association for Information Systems, Decision Support Systems, Expert Systems with Applications, Government Information Quarterly, Information \& Management, International Journal of Electronic Government Research, and MIS Quarterly. Conference proceedings regularly including UTAUT material included the Americas Conference of Information Systems, the European Conference of Information Systems, the Hawaii International Conference on System Sciences, and the Southern Association for Information Systems Conference. The keywords associated with these 174 articles were noted, and in keeping with the works of Lee et al. (2003) and Legris et al. 
(2003) in their comparable studies works examining the use of TAM, the articles were analysed in terms of a series of characteristics including types of relationships found between model constructs, external variables, limitations of studies, and methodological details.

\section{Research Findings}

Many different researchers with different research intentions and subjects of focus have conducted UTAUT studies by applying a variety of research methodologies in different environments. This diverse body of work has seen numerous new constructs being incorporated into the original theory, with UTAUT being blended with other theoretical models, and on occasion, a re-specification of the underlying relationships between UTAUT variables. These research papers were published in journals and conferences in diverse streams of study, and in line with the findings of Lee et al. (2003) in their study of TAM, were seen to have drawn the attention of both researchers and practitioners. This section presents an analysis of these UTAUT studies by examining a number of variables including most productive authors, universities/institutions and authors, most productive departments, university affiliation according to country, sources of primary data by country, authors' academic backgrounds, publications frequency, number of authors, publication outlets for UTAUT researchers, keyword analysis, types of systems examined, research subjects, relationships between major UTAUT variables, weight analysis, relationship of external variables with UTAUT constructs, and most frequently used external variables.

\subsection{Demographic Characteristics}

\subsubsection{Most Productive Authors}

Our analysis of the most prolific authors revealed that 494 authors contributed to the 174 UTAUT studies. Table 1 illustrates the 11 authors who have published three or more papers. These authors published a total of 23 of the 174 research papers, and thus there currently appears to be no dominant group of authors as such. This result is quite different to that obtained by Lee et al. (2003) in their study of TAM, where almost 50\% of research papers considered were provided by a group of 11 authors. This clearly indicates that studies on UTAUT are not yet dominated by any group of highly productive individuals, publications currently being scattered across a large number of authors each contributing fewer articles.

Table 1

Most Productive Authors

\begin{tabular}{|l|l|c|}
\hline Prolific Authors & University & \# of Articles \\
\hline Gang Liu & Renmin University of China & 4 \\
\hline Susan A. Brown & University of Arizona & 4 \\
\hline Vishanth Weerakkody & Brunel University & 4 \\
\hline Yaobin Lu & Huazhong University of Science and Technology & 4 \\
\hline Viswanath Venkatesh & University of Arkansas & 4 \\
\hline Bram Pynoo & Ghent University & 4 \\
\hline Cheng Qian & Renmin University of China & 3 \\
\hline Dehua He & Huazhong University of Science and Technology & 3 \\
\hline Dong Cheng & Renmin University of China & 3 \\
\hline Paul H.P. Yeow & Multimedia University & 3 \\
\hline Shafi Al-Shafi & Brunel University & 3 \\
\hline
\end{tabular}

\subsubsection{Contributing Universities/Institutions and Authors}


Table 2 illustrates 18 universities/institutions associated with the highest combinations of numbers of papers published and associated non-adjusted counts of contributing authors/coauthors. Renmin University in China appears at the top of this list, with five published outputs and a non-adjusted author count of 16. This corresponds with Table 1, which includes three individuals from Renmin University in the list of most productive authors. Ghent University appears in second place, with four publications and a non-adjusted author count of 25 , the high author count in this case arising from a single paper with seven authors and three more with six authors each. Surprisingly, U.S Universities (including the University of Arkansas, the University of Nevada, and the University of Georgia) appear relatively low on this list (in comparison with other reviews of research) in terms of the number of papers produced.

Table 2

Publications by University/Institution

\begin{tabular}{|l|c|c|}
\hline University/Institution & \# of Papers & $\begin{array}{c}\text { Author Count } \\
\text { (Non-adjusted) }\end{array}$ \\
\hline Renmin University of China & 5 & 16 \\
\hline Ghent University & 4 & 25 \\
\hline Brunel University & 4 & 11 \\
\hline Multimedia University & 4 & 8 \\
\hline Huazhong University of Science and Technology & 4 & 6 \\
\hline National Changhua University of Education & 4 & 5 \\
\hline University of St. Gallen & 3 & 9 \\
\hline University of Cape Town & 3 & 5 \\
\hline University of Macedonia & 3 & 5 \\
\hline National Chung Cheng University & 2 & 7 \\
\hline Korea Advance Institute of Science and Technology & 2 & 6 \\
\hline University of Nevada & 2 & 6 \\
\hline University of North Texas & 2 & 6 \\
\hline University of Arkansas & 2 & 5 \\
\hline University of Twente & 2 & 5 \\
\hline University of Georgia & 1 & 7 \\
\hline Chinese Academy of Sciences & 1 & 6 \\
\hline University of Technology Sydney & 1 & 5 \\
\hline
\end{tabular}

\subsubsection{Most Productive Departments}

Table 3 illustrates the home departments of the authors or co-authors who have contributed to publishing papers on UTAUT. By far the majority of authors belonged to departments related to the business, management, information systems and technology fields, whereas a far smaller group belonged to departments including journalism and mass communication, and radiology and medical imaging. These departments (Table 3) accounted for 145 of the total of 328 contributing departments.

Table 3

Most Productive Departments

\begin{tabular}{|l|c|}
\hline Department/School & $\#$ \\
\hline Department of Information Management / Systems & 57 \\
\hline School /college of Business / Management / Business School & 55 \\
\hline Department of Radiology / Medical Imaging & 15 \\
\hline Faculty of Psychology and Educational Sciences & 7 \\
\hline
\end{tabular}


\begin{tabular}{|l|l|} 
College of Journalism and Mass Communication & 7 \\
\hline Department of Telecommunication, Information Studies and Media & 4 \\
\hline
\end{tabular}

\subsubsection{University Affiliation According to Country}

Table 4 presents the 20 countries whose universities contributed the most UTAUT research publications. Of a total of 494 occurrences from 36 distinct countries, and 219 unique universities, the highest proportion of work was produced from universities in the USA (\#140, 28\%), followed by some way behind by Taiwan (\#46), China (\#43), the UK (\#38), Belgium (\#28), Malaysia (\#26), and Australia (\#21). The low ranking of USA-based universities in Table 2 and their top ranking in Table 4 is explained by the diffusion of UTAUT research across a large number of institutions in the USA, each producing a comparatively low number of publications. Universities in numerous countries contributed three or fewer studies, including Cyprus, Ethiopia, Hong Kong, Peru, Saudi Arabia, and Tanzania, which contributed one study each - these are not listed in Table 4 due to space limitations.

Table 4

University Affiliation According to Country

\begin{tabular}{|l|c|l|c|}
\hline Researchers' Originating Country & $\#$ & Researchers' Originating Country & $\#$ \\
\hline USA & 140 & Greece & 13 \\
\hline Taiwan & 46 & South Korea & 10 \\
\hline China & 43 & Italy & 9 \\
\hline UK & 39 & South Africa & 9 \\
\hline Belgium & 28 & Canada & 7 \\
\hline Malaysia & 26 & Switzerland & 7 \\
\hline Australia & 21 & Sweden & 6 \\
\hline Netherlands & 18 & Singapore & 4 \\
\hline Germany & 16 & Slovenia & 4 \\
\hline Finland & 16 & Uganda & 4 \\
\hline
\end{tabular}

\subsubsection{Sources of Primary Data by Country}

Our findings (Table 5) reveal that published UTAUT research has been based on primary data captured in 41 countries. By far the most popular source of primary data has been the USA (\#45), followed some way behind by China (\#19), Taiwan (\#17), and then Malaysia (\#10), Australia (\#8), India (\#6), Belgium (\#5), and Saudi Arabia (\#5). Countries such as Hong Kong, Italy, Peru, Sweden, Tanzania, Thailand, and the UK were used only twice to collect primary data, and a large number of countries - including Austria, Bangladesh, Cyprus, Denmark, Ethiopia, Indonesia, Kuwait, Lithuania, Pakistan, Philippines, Portugal, Romania, Slovenia, South Korea, Sri Lanka, Switzerland, and Uganda - were each used only once to collect such data.

Table 5

Most Used Countries for Primary Data Collection

\begin{tabular}{|l|c|l|c|}
\hline Country & $\#$ & Country & $\#$ \\
\hline USA & 45 & Germany & 4 \\
\hline China & 19 & Canada & 3 \\
\hline Taiwan & 17 & Greece & 3 \\
\hline Malaysia & 10 & Jordan & 3 \\
\hline Australia & 8 & Netherlands & 3 \\
\hline India & 6 & Qatar & 3 \\
\hline
\end{tabular}




\begin{tabular}{|l|c|l|c|} 
Belgium & 5 & Singapore & 3 \\
\hline Saudi Arabia & 5 & South Africa & 3 \\
\hline Finland & 5 & Total & 145 \\
\hline
\end{tabular}

\subsubsection{Authors' Academic Backgrounds}

In order to examine the academic background of the authors, their associated organisations were divided into three major divisions; academics, public sector, and industry. The findings suggest a summary of the results - unsurprisingly 98\% (\#484) of authors had an academic background, whereas only four belonged to the public sector, and six were from industry.

\subsubsection{Frequency of Publication}

This analysis displays the number of publications of UTAUT work appearing between 2004 and June 2011. The findings indicate that the number of publications has generally increased year upon year since the appearance of the original article such as four articles each in 2004 and 2005, nine articles in 2006, 16 articles 2007, 35 articles in 2008, 46 articles in 2009, and the highest 48 articles in 2010, with a significant increase in numbers since 2008 and before a complete trend for the further years was analysed. We suggest that this upward trend will continue and future years will see a further increase in the number of UTAUT-related papers published.

\subsubsection{Number of Authors}

The findings on the number of authors reveal the frequency of UTAUT research publications being authored and co-authored by between one and seven authors. Two authors created the largest 61 research papers, whereas two papers were published by a group of seven authors. Moreover, 16 articles were single authored, three authors authored 54 articles, four authors authored 28 articles, five authors authored five articles, and six authors authored eight articles.

\subsubsection{Publication Outlets for UTAUT Researchers}

Table 6 illustrates 20 outlets that have each published two or more UTAUT research papers. Numerous conferences have published UTAUT-research, including the Americas Conference of Information Systems (\#6), the European Conference of Information Systems (\#5), the IEEE Conference (\#4), and the Hawaii International Conference on System Sciences (\#4) among others. Similarly, a series of high-ranking internationally recognized journals including Expert Systems with Applications (\#3), Government Information Quarterly (\#3), Information \& Management (\#3), and MIS Quarterly (\#3) also appear in Table 6, indicating their willingness to accept and publish UTAUT-based research. In addition to the conferences and journals appearing in Table 6, a further 111 outlets each published one paper. This suggest that the publishing landscape for UTAUT researchers is currently quite diverse and widespread, and this is quite unlike the findings of Lee et al. (2003) in their study of TAM which found TAM outputs to be concentrated across a relatively small number of journals such as MIS Quarterly, Information \& Management, Information Systems Research, and the Journal of Management Information Systems among others. 
Publishers of UTAUT Research Articles

\begin{tabular}{|l|l|}
\hline Journal / Conference Name & $\#$ \\
\hline Americas Conference on Information Systems & 6 \\
\hline European Conference on Information Systems & 5 \\
\hline Computers in Human Behavior & 4 \\
\hline Computers \& Education & 4 \\
\hline IEEE Conference & 4 \\
\hline Hawaii International Conference on System Sciences & 4 \\
\hline Communications of the Association for Information Systems & 3 \\
\hline Expert Systems with Applications & 3 \\
\hline Government Information Quarterly & 3 \\
\hline Information \& Management & 3 \\
\hline International Journal of Electronic Government Research & 3 \\
\hline MIS Quarterly & 3 \\
\hline Decision Support Systems & 2 \\
\hline Communications of the IBIMA & 2 \\
\hline DIGIT 2009 & 2 \\
\hline International Journal of Accounting Information Systems & 2 \\
\hline International Journal of Medical Informatics & 2 \\
\hline Southern Association for Information Systems Conference & 2 \\
\hline WEBIST & 2 \\
\hline European Journal of Information Systems & 2 \\
\hline
\end{tabular}

\subsection{IS Research Topics and Types of Systems Examined}

\subsubsection{Keyword Analysis}

Table 7 lists the 30 most frequently used keywords (each occurring three or more times across 174 studies) in UTAUT research. These keywords account for 272 of the overall total of 739 keyword occurrences of the 450 unique keywords identified. As expected, "Unified Theory of Acceptance and Use of Technology"/"UTAUT" (\#79) appeared most often, followed by "Technology Acceptance" (\#27), "Technology Acceptance Model” (\#20), "Adoption" (\#13), “Technology Adoption” (\#13), "E-Government” (\#11), "User Acceptance" (\#11), and "Trust" (\#9) as some of the other more frequently utilized keywords. In addition, various constructs of UTAUT such as "performance expectancy", "effort expectancy", and "social influence" were also among the keywords appearing three or more times. The regular appearance of certain words and terms such as "acceptance", "adoption", "Internet banking", "end user", "electronic government", "electronic commerce" and "mobile commerce", "structural equation modelling" and "partial least squares" gives the suggestion that many UTAUT studies are focused on investigating the acceptance, adoption, and use of technology in various forms of banking, government services and commerce, and are employing widely utilized analysis methods such as SEM and PLS. However, a large body of keywords (\#418) appear once (\#369) or twice (\#49), and these aspects are worthy of further exploration.

Table 7

Most Frequently Used Keywords (Approach from Dwivedi et al., 2008)

\begin{tabular}{|l|l|l|l|}
\hline Keywords & $\#$ & Keywords & $\#$ \\
\hline Unified Theory of Acceptance and Use of Technology & 79 & Acceptance & 4 \\
\hline Technology Acceptance & 27 & Performance Expectancy & 4 \\
\hline Technology Acceptance Model & 20 & Saudi Arabia & 4 \\
\hline Adoption & 13 & Structural Equation Modelling & 4 \\
\hline Technology Adoption & 13 & M-Commerce & 4 \\
\hline
\end{tabular}




\begin{tabular}{|c|c|c|}
\hline E-Government & 11 E-Commerce & 3 \\
\hline User Acceptance & 11 Ease of Use & \\
\hline Trust & \begin{tabular}{|l|l|}
9 & Effort Expectancy \\
\end{tabular} & 3 \\
\hline Internet Banking & \begin{tabular}{l|l}
7 & Evaluation \\
\end{tabular} & 3 \\
\hline E-Learning & $\begin{array}{ll}6 & \text { Gender } \\
\end{array}$ & \\
\hline Intention To Use & $\begin{array}{ll}6 & \text { Information Systems } \\
\end{array}$ & \\
\hline Developing Countries & \begin{tabular}{c|c}
5 & Information Technology
\end{tabular} & \\
\hline Partial Least Squares & \begin{tabular}{l|l}
5 & End User
\end{tabular} & \\
\hline Perceived Risk & $\begin{array}{ll}5 & \text { Mobile Business } \\
\end{array}$ & 3 \\
\hline Social Influence & \begin{tabular}{l|l}
5 & Usability \\
\end{tabular} & 3 \\
\hline
\end{tabular}

\subsubsection{Systems Examined}

Over 98 different types of system were examined in the articles under analysis, being classified into the same four categories originally defined by Lee et al. (2003) in their review of TAM research: communication systems (25), general-purpose systems (90), office systems (11), and specialized business systems (48). General purpose systems were most frequently examined, and office systems the least. As per the work of Lee et al. (2003), general-purpose systems include Windows, personal computers, microcomputers, workstations, the Internet, and other general-purpose computer facilities. Communication systems included mobilebased technology, kiosk systems, automated feedback systems, instant messaging, and other systems primarily used for communications. Mobile technology was the most widely examined technology for communication systems. Office systems include applications that are commonly found in the office environment (such as desktop applications, database and query systems), whereas specialized systems included systems such as e-procurement systems, ERP systems, and e-voting systems. Table 8 presents details of systems included within each category along with the associated publications.

Table 8

Systems Used in UTAUT Studies (Approach from Lee et al., 2003)

\begin{tabular}{|c|c|c|c|}
\hline Type & \# of IS & ISs for Each Category & Associated Publication(s) \\
\hline \multirow{15}{*}{$\begin{array}{l}\text { Communication } \\
\text { Systems }\end{array}$} & \multirow{15}{*}{$\begin{array}{c}25 \\
(14 \%)\end{array}$} & Mobile Banking (4) & $\begin{array}{l}\text { Barati and Mohammadi (2009), de Silva } \\
\text { and Ratnadiwakara (2009), Luo et al. } \\
\text { (2010), Zhou et al. (2010) }\end{array}$ \\
\hline & & Robot System (2) & $\begin{array}{l}\text { BenMessaoud et al. (2011), Heerink et } \\
\text { al. (2009) }\end{array}$ \\
\hline & & Mobile Podcasting (1) & Ho and Chou (2009) \\
\hline & & M-Coupon System (1) & Jayasingh and Eze (2009) \\
\hline & & Information Kiosk (2) & $\begin{array}{l}\text { Johari et al. (2010), Wang and Shih } \\
(2009)\end{array}$ \\
\hline & & Mobile Internet Application (1) & Kourouthanassis et al. (2010) \\
\hline & & Instant Messaging (1) & Lin et al. (2004) \\
\hline & & Mobile Commerce (2) & $\begin{array}{l}\text { Qingfei et al. (2008), Tan and } \mathrm{Wu} \\
(2010)\end{array}$ \\
\hline & & Mobile Technology (2) & Park et al. (2007), Song and Han (2009) \\
\hline & & Mobile Phone/Internet (3) & $\begin{array}{l}\text { van Biljon and Kotze (2008), van Biljon } \\
\text { and Renaud (2008), Wang et al. (2010) }\end{array}$ \\
\hline & & Mobile Shopping Services (1) & Yang (2010) \\
\hline & & Mobile Advertising (1) & He and Lu (2007) \\
\hline & & 3G Mobile Communication (2) & Wu et al. (2007), Wu et al. (2008) \\
\hline & & Digital Television (1) & Sapio et al. (2010) \\
\hline & & Automated Feedback System (1) & Debuse et al. (2008) \\
\hline General Purpose & 90 & Internet/Online Banking (10) & AbuShanab et al. (2010), AbuShanab \\
\hline
\end{tabular}




\begin{tabular}{|c|c|c|c|}
\hline \multirow[t]{26}{*}{ Systems } & \multirow[t]{26}{*}{$(52 \%)$} & & $\begin{array}{l}\text { and Pearson (2007), Abu-Shanab and } \\
\text { Pearson (2009), Al-Somali et al. (2009), } \\
\text { Cheng et al. (2008a), Cheng et al. } \\
\text { (2008b), Cheng et al. (2008c), Liu et al. } \\
\text { (2008), YenYuen and Yeow (2009), } \\
\text { Yeow et al. (2008) }\end{array}$ \\
\hline & & Information System/Technology (14) & $\begin{array}{l}\text { Al-Gahtani et al. (2007), Al-Rajhi et al. } \\
\text { (2010), Bandyopadhyay and and } \\
\text { Bandyopadhyay (2010), Brown and } \\
\text { Venkatesh (2005), Dadayan and Ferro } \\
\text { (2005), Diaz and Loraas (2010), Laumer } \\
\text { et al. (2010), Neufeld et al. (2007), } \\
\text { Pahnila et al. (2011), Schaper and } \\
\text { Pervan (2006), Sharma and Citurs } \\
\text { (2004), Suhendra et al. (2009), Teo } \\
\text { (2011), Venkatesh et al. (2008) }\end{array}$ \\
\hline & & E-Government Services (9) & $\begin{array}{l}\text { Al-Shafi and Weerakkody (2009), Al- } \\
\text { Shafi and Weerakkody (2010), Al-Sobhi } \\
\text { et al. (2011), AlAwadhi and Morris } \\
\text { (2011), Chan et al. (2010), Hung et al. } \\
\text { (2007), Sahu and Gupta (2007), Suki } \\
\text { and Ramayah (2010), Weerakkody et al. } \\
\text { (2009) }\end{array}$ \\
\hline & & E-Filing System (3) & $\begin{array}{l}\text { Ambali (2009), Carter and Schaupp } \\
\text { (2009), Schaupp et al. (2010) }\end{array}$ \\
\hline & & Tablet PCs (1) & Anderson et al. (2006) \\
\hline & & Internet/Intranet Technology (6) & $\begin{array}{l}\text { Barnes and Vidgen (2009), Dasgupta } \\
\text { and Gupta (2010), Foon and Fah (2011), } \\
\text { Huang et al. (2010), Niehaves and } \\
\text { Plattfaut (2010), van Dijk et al. (2008) }\end{array}$ \\
\hline & & E-Quality (2) & $\begin{array}{l}\text { Cody-Allen and Kishore (2006), } \\
\text { Samoutis et al. (2008) }\end{array}$ \\
\hline & & ICT (5) & $\begin{array}{l}\text { Cornacchia et al. (2008), Gupta et al. } \\
\text { (2008), Im et al. (2008), Schaper and } \\
\text { Pervan (2004), Verhoeven et al. (2010) }\end{array}$ \\
\hline & & E-Readiness (1) & Dada (2006) \\
\hline & & Knowledge Management System (3) & $\begin{array}{l}\text { He and Wei (2009), Jalaldeen et al. } \\
(2009), \operatorname{Li}(2010)\end{array}$ \\
\hline & & Security Information System (1) & Johnston and Warkentin (2010) \\
\hline & & Web-based Virtual M-Learning System (8) & $\begin{array}{l}\text { Chiu and Wang (2008), Jong and Wang } \\
\text { (2009), Keller et al. (2007), Nistor et al. } \\
\text { (2010), Sumak et al. (2010), Tsai et al. } \\
\text { (2009), van Raaij and Schepers (2008), } \\
\text { Wang et al. (2009) }\end{array}$ \\
\hline & & Software Technologies (1) & Koh et al. (2010) \\
\hline & & Podcasting (1) & Lee and Lin (2008) \\
\hline & & Activity Based Costing (1) & Lee et al. (2010a) \\
\hline & & Smart Products (1) & Mayer et al. (2011) \\
\hline & & Wi-Fi System (1) & Udeh (2008) \\
\hline & & E-Commerce/Mobile Commerce (2) & Uzoka (2008), Zhou (2008) \\
\hline & & World Wide Web (1) & Pavon and Brown (2010) \\
\hline & & Web $2.0(1)$ & Payne (2008) \\
\hline & & Educational Technology System (1) & Wu et al. (2010) \\
\hline & & Location-Based Services (1) & Xu and Gupta (2009) \\
\hline & & Collaboration Technology (1) & Brown et al. (2010) \\
\hline & & E-Health Services/Health IS (2) & $\begin{array}{l}\text { Chiu and Eysenbach (2010), Fitterer et } \\
\text { al. (2010) }\end{array}$ \\
\hline & & Social Media (1) & Curtis et al. (2010) \\
\hline & & Open Access (1) & Dulle and Minishi-Majanja (2011) \\
\hline
\end{tabular}




\begin{tabular}{|c|c|c|c|}
\hline & & Mobile Business (2) & He and Lu (2008), He et al. (2007) \\
\hline & & Mobile Services (2) & $\begin{array}{l}\text { Carlsson et al. (2006), Koivumaki et al. } \\
(2008)\end{array}$ \\
\hline & & Educational Portal (2) & $\begin{array}{l}\text { Maldonado et al. (2009), Maldonado et } \\
\text { al. (2011) }\end{array}$ \\
\hline & & Web-Based Technology (1) & Or et al. (2011) \\
\hline & & Digital Learning (1) & Pynoo et al. (2011) \\
\hline & & Cross-Cultural Information Retrieval (1) & Taksa and Flomenbaum (2009) \\
\hline & & Problem Solving Models (1) & Richardson et al. (2009) \\
\hline & & Websites (1) & van Schaik (2009) \\
\hline \multirow{10}{*}{ Office Systems } & \multirow{10}{*}{$11(6 \%)$} & Accounting Information System (1) & Aoun et al. (2010) \\
\hline & & Computer-Assisted Audit Techniques (2) & $\begin{array}{l}\text { Curtis and Payne (2008), Mahzan and } \\
\text { Lymer (2008) }\end{array}$ \\
\hline & & Remote Desktop Application (1) & Hutchison and Bekkering (2009) \\
\hline & & Reference Databases (1) & Avdic and Eklund (2010) \\
\hline & & Decision Making Trial and Evaluation (1) & Lee et al. (2010b) \\
\hline & & Computer Graphics Technology (1) & Shamsuddin (2009) \\
\hline & & Peer-to-Peer Academic Networks (1) & Tavares and Amarel (2010) \\
\hline & & Computer Based Assessment Model (1) & Terzis and Economides (2011) \\
\hline & & EHR Query System (1) & Huser et al. (2010) \\
\hline & & Software Cost Estimation (1) & Yang et al. (2008) \\
\hline \multirow{29}{*}{$\begin{array}{l}\text { Specialized } \\
\text { Business } \\
\text { Systems }\end{array}$} & \multirow{29}{*}{$\begin{array}{c}48 \\
(28 \%)\end{array}$} & Biometrics Authentication System (1) & Al-Harby et al. (2010) \\
\hline & & Medical Teleconferencing Application (1) & Biemans et al. (2005) \\
\hline & & iBrainz Technology (1) & Butler and Richardson (2008) \\
\hline & & Water Treatment Technology (1) & Cabral et al. (2009) \\
\hline & & Weblog Technology (2) & $\begin{array}{l}\text { Chen et al. (2008), Li and Kishore } \\
(2006)\end{array}$ \\
\hline & & Medical Support System (1) & Coss (2009) \\
\hline & & Micro Blogging (1) & Gunther et al. (2009) \\
\hline & & Electronic HRM (1) & Heikkila and Smale (2010) \\
\hline & & Electronic Medical Record System (4) & $\begin{array}{l}\text { Chisolm et al. (2010), Hennington et al. } \\
(2009) \text {, Trimmer et al. (2008), Wills et } \\
\text { al. (2008) }\end{array}$ \\
\hline & & Telemedicine (1) & Hailemariam et al. (2010) \\
\hline & & Hybrid Media Application (1) & Louho et al. (2006) \\
\hline & & Motes (1) & Lubrin et al. (2006) \\
\hline & & Course Management Software (1) & Marchewka et al. (2007) \\
\hline & & Tax Software System (2) & McLeod et al. (2009a, 2009b) \\
\hline & & Enterprise Mashup System (1) & Pahlke and Beck (2009) \\
\hline & & Personal Health Record System (1) & Randeree (2009) \\
\hline & & E-Ordering Application (1) & Reunis and Santema (2005) \\
\hline & & Electronic Procurement System (2) & $\begin{array}{l}\text { Benslimane et al. (2004), Sambasivan et } \\
\text { al. (2010) }\end{array}$ \\
\hline & & Enterprise Resource Planning Systems (1) & Seymour et al. (2007) \\
\hline & & Hybrid/Digital Library (2) & $\begin{array}{l}\text { Nov and Ye (2009), Tibenderana et al. } \\
(2010)\end{array}$ \\
\hline & & $\begin{array}{l}\text { Picture Archiving and Communication } \\
\text { System (3) }\end{array}$ & $\begin{array}{l}\text { Duyck et al. (2008), Duyck et al. (2010), } \\
\text { Pynoo et al. (2008) }\end{array}$ \\
\hline & & ATM and Transit Application (1) & Yeow and Loo (2009) \\
\hline & & ERP System (1) & Huang and Wang (2009) \\
\hline & & $\begin{array}{l}\text { Customer Relationship Management (CRM) } \\
\text { Systems (1) }\end{array}$ & Pai and Tu (2011) \\
\hline & & Audience Counts and Reporting System (1) & Pappas and Volk (2007) \\
\hline & & Smart Phone Online Application (1) & Shi (2009) \\
\hline & & MVNO Services (1) & Shin (2010) \\
\hline & & Telehospice (1) & Whitten et al. (2009) \\
\hline & & Remote Electronic Voting Systems (1) & Yao and Murphy (2007) \\
\hline
\end{tabular}




\begin{tabular}{|l|l|l|}
\hline & Hospital Information System (1) & Aggelidis and Chatzoglou (2009) \\
\hline & Speech Recognition System (1) & Alapetite et al. (2009) \\
\hline Clinical Decision Support System (1) & Chang et al. (2007) \\
\hline & Online Auctions (1) & Chiu et al. (2010) \\
\hline Health Information Technology (1) & Kijsanayotin et al. (2009) \\
\hline Mobile Wallet (1) & Shin (2009) \\
\hline Forecasting Support System (1) & Lee et al. (2007) \\
\hline Smartcard Application (1) & Loo et al. (2009) \\
\hline Human Computer Interaction Tool (1) & Oshlyansky et al. (2007) \\
\hline Recommender System (1) & van Setten et al. (2006) \\
\hline
\end{tabular}

\subsection{Methodological Analysis}

\subsubsection{Research Methodology Used}

Our findings (see Table 9) revealed that only 18 out of 174 studies were longitudinal in nature, the majority of studies (\#135) using a cross-sectional approach. As far as research methodologies were concerned, survey instrument (\#155) was most commonly used, followed some way behind by a collection of lesser-used techniques including interview (\#12), case study (\#4), field study (\#3), laboratory experiment (\#3), and literature study (\#1). Field study (\#3) is currently one of the least used methodologies in our research, unlike Lee et al.'s (2003) examination of TAM research in which field study was seen to be the most common methodology.

Table 9

Research Methodologies (Approach from Lee et al., 2003)

\begin{tabular}{|c|c|c|}
\hline Methodology & Details & Example Reference(s) \\
\hline \multirow{3}{*}{$\begin{array}{l}\text { Research } \\
\text { Approach }\end{array}$} & Longitudinal (18) & $\begin{array}{l}\text { Brown and Venkatesh (2005), Heerink et al. } \\
\text { (2009) }\end{array}$ \\
\hline & Cross-Sectional (135) & $\begin{array}{l}\text { Al-Somali et al. (2009), Johnston and Warkentin } \\
(2010)\end{array}$ \\
\hline & Exploratory Study (21) & $\begin{array}{l}\text { Al-Rajhi et al. (2010), Cody-Allen and Kishore } \\
\text { (2006) }\end{array}$ \\
\hline \multirow{6}{*}{ Methodology } & Survey (155) & $\begin{array}{l}\text { Kourouthanassis et al. (2010), Venkatesh et al. } \\
\text { (2008) }\end{array}$ \\
\hline & Interview (12) & Heikkila and Smale (2010), Li (2010) \\
\hline & Case Study (4) & Samoutis et al. (2008), Trimmer et al. (2008) \\
\hline & Field Study (3) & Brown et al. (2010), Chen et al. (2008) \\
\hline & Laboratory Experiment (3) & Al-Harby et al. (2010), Lee et al. (2007) \\
\hline & Literature Study (1) & $\mathrm{He}$ and $\mathrm{Lu}(2007 \mathrm{~b})$ \\
\hline \multirow{12}{*}{ Analysis Method } & Structural Equation Modelling (45) & Laumer et al. (2010), Wang et al. (2010) \\
\hline & Regression Analysis (42) & Sapio et al. (2010), van Dijk et al. (2008) \\
\hline & PLS Analysis (27) & Koh et al. (2010), Lin et al. (2004) \\
\hline & Confirmatory Factor Analysis (15) & Wu et al. (2010), Xu and Gupta (2009) \\
\hline & Factor Analysis (13) & Curtis et al. (2010), Fitterer et al. (2010) \\
\hline & ANOVA (12) & Cornacchia et al. (2008), Shamsuddin (2009) \\
\hline & Correlation Analysis (6) & Cornacchia et al. (2008), Heerink et al. (2009) \\
\hline & Cluster Analysis (1) & Benslimane et al. (2004) \\
\hline & Content Analysis (1) & BenMessaoud et al. (2011) \\
\hline & Descriptive Analysis (1) & Huang and Wang (2009) \\
\hline & ANCOVA (1) & van Schaik (2009) \\
\hline & OLS (1) & Ambali (2009) \\
\hline
\end{tabular}




\begin{tabular}{|l|l|l|}
\hline \multirow{5}{*}{} & AVE Analysis (1) & Yao and Murphy (2007) \\
\cline { 2 - 3 } & Invariance Analysis (1) & Li and Kishore (2006) \\
\cline { 2 - 3 } & Structural Model (1) & Chan et al. (2010) \\
\cline { 2 - 3 } & Path Analysis (1) & Suhendra et al. (2009) \\
\cline { 2 - 3 } & Secondary Analysis (1) & Or et al. (2011) \\
\hline \multirow{5}{*}{ Analysis Tool } & SPSS (30) & Jayasingh and Eze (2009), Pynoo et al. (2008) \\
\cline { 2 - 3 } & AMOS (12) & Schaupp et al. (2010), Shin (2010) \\
\cline { 2 - 3 } & LISREL (8) & Song and Han (2009), Zhou et al. (2010) \\
\cline { 2 - 3 } & PLS Graph 3.0 (7) & van Raaij and Schepers (2008), Wu et al. (2010) \\
\cline { 2 - 3 } & Smart PLS 2.0 (3) & Chan et al. (2010), Laumer et al. (2010) \\
\cline { 2 - 3 } & Build 1126 (1) & Brown et al. (2010) \\
\cline { 2 - 3 } & SAS (1) & Tsai et al. (2009) \\
\cline { 2 - 3 } & SQL (1) & Huser et al. (2010) \\
\cline { 2 - 3 } & Visual Basic 6.0 (1) & van Schaik (2009) \\
\hline
\end{tabular}

Survey instruments were commonly used in different forms such as questionnaire survey, telephone survey, and online or Web-based survey. Much data analysis involved structural equation modelling (\#45) using software such as AMOS (\#12), PLS (\#11), and LISREL (\#8) or regression analysis (\#42) using SPSS (\#30). Currently, SPSS is the most commonly used data analysis tool, whereas Lee et al.'s (2003) study on TAM revealed the use of LISREL to be predominant.

\subsubsection{Research Subjects}

Table 10 illustrates that the UTAUT studies can be divided in four broad categories according to user type or alternative source of data, viz: general users, professionals, students, and literature studies. The studies of Brown et al. (2010), McLeod et al. (2009a), Tibenderana et al. (2010), and Zhou et al. (2010) used more than one user type for data collection, thus accounting for the total of 178 studies.

Table 10

Research Subjects (Approach from Lee et al., 2003)

\begin{tabular}{|l|c|l|}
\hline User Type & \# of Studies & Example Studies \\
\hline General Users & 63 & Johnston and Warkentin (2010), Park et al. (2007) \\
\hline Professionals & 74 & Pai and Tu (2011), Pynoo et al. (2008) \\
\hline Students & 40 & Maldonado et al. (2011), Tsai et al. (2009) \\
\hline Literature Study & 1 & He and Lu (2007b) \\
\hline
\end{tabular}

\subsection{Internal Variable Analysis}

\subsubsection{Relationships between Major UTAUT Variables}

UTAUT's six main variables are: Performance Expectancy (PE), Effort Expectancy (EE), Social Influence (SI), Behavioural Intention (BI), and Usage Behaviour (UB), BI being both an independent and dependent variable. A total of 102 of the 174 studies were quantitative in nature and presented quantitative representations of the relationships between constructs. Of these 102 studies, 32 made use of UTAUT more than once in the same study due to different models, user types, or time span implementations resulting in a total of 159 different occurrences of the relationships between corresponding variables. As shown in Table 11, no single study was seen to support all UTAUT relationships (indeed, some studies did not examine all relationships, and yet others examined variations in the original relationships), but all UTAUT relationships are supported by at least one study. The results of this analysis are summarised in Table 11. 
Table 11

Results of Examining Relationships (Approach from Legris et al., 2003)

\begin{tabular}{|c|c|c|c|c|c|c|}
\hline Study & PE-BI & EE-BI & SI-BI & FC-BI & FC-U & BI-U \\
\hline AbuShanab and Pearson (2007) & Yes & Yes & Yes & $\mathrm{x}$ & $\mathrm{x}$ & $\mathrm{x}$ \\
\hline AbuShanab et al. (2010) & Yes & Yes & Yes & $\mathrm{x}$ & $\mathrm{x}$ & $\mathrm{x}$ \\
\hline Aggelidis and Chatzoglou (2009) & $\mathrm{x}$ & $\mathrm{x}$ & Yes & Yes & $\mathrm{x}$ & $\mathrm{x}$ \\
\hline \multicolumn{7}{|l|}{ AlAwadhi and Morris (2011) } \\
\hline T1- 4 Weeks & $\mathrm{Yes}^{\mathrm{E}}$ & $\mathrm{Yes}^{\mathrm{E}}$ & Yes & $\mathrm{x}$ & $\mathrm{x}$ & $\mathrm{x}$ \\
\hline T2- 3 Months & Yes & Yes & $\mathrm{x}$ & $\mathrm{x}$ & $\mathrm{x}$ & $\mathrm{x}$ \\
\hline T3- Future & Yes & Yes & $\mathrm{x}$ & $\mathrm{x}$ & $\mathrm{x}$ & $\mathrm{x}$ \\
\hline Impact on Use Behavior & $\mathrm{X}$ & $\mathrm{x}$ & $\mathrm{X}$ & $\mathrm{x}$ & Yes & Yes \\
\hline \multicolumn{7}{|l|}{ Al-Gahtani et al. (2007) } \\
\hline Model 1 - Without Moderating Variables & Yes & Yes & $\mathrm{x}$ & $\mathrm{x}$ & Yes & Yes \\
\hline Model 2 - With Moderating Variables & Yes $^{\mathrm{AE}}$ & $\mathrm{No}^{\mathrm{AE}}$ & $\mathrm{x}$ & $\mathrm{x}$ & $\mathrm{No}^{\mathrm{AE}}$ & $\mathrm{Yes}^{\mathrm{AE}}$ \\
\hline Al-Shafi and Weerakkody (2009) & Yes & Yes & Yes & $\mathrm{x}$ & $\mathrm{x}$ & $\mathrm{X}$ \\
\hline Al-Shafi and Weerakkody (2010) & No & Yes & Yes & $\mathrm{x}$ & $\mathrm{x}$ & $\mathrm{x}$ \\
\hline Al-Sobhi et al. (2011) & No & Yes & Yes & $\mathrm{x}$ & Yes & No \\
\hline Ambali (2009) & $\mathrm{x}$ & $\mathrm{x}$ & $\mathrm{x}$ & $\mathrm{x}$ & Yes & $\mathrm{x}$ \\
\hline \multicolumn{7}{|l|}{ Anderson et al. (2006) } \\
\hline Model-1 & $\mathrm{x}$ & $\mathrm{X}$ & $\mathrm{x}$ & $\mathrm{x}$ & No & $\mathrm{x}$ \\
\hline Model-2 & $\mathrm{x}$ & $\mathrm{x}$ & $\mathrm{x}$ & $\mathrm{x}$ & No & $\mathrm{x}$ \\
\hline Aoun et al. (2010) & Yes & Yes & No & $\mathrm{x}$ & Yes & Yes \\
\hline \multicolumn{7}{|l|}{ Bandyopadhyay and Bandyopadhyay (2010) } \\
\hline India & $\mathrm{Yes}^{\mathrm{M}}$ & $\mathrm{Yes}^{\mathrm{M}}$ & $\mathrm{Yes}^{\mathrm{M}}$ & $\mathrm{x}$ & $\mathrm{x}$ & $\mathrm{x}$ \\
\hline USA & $\mathrm{Yes}^{\mathrm{M}}$ & $\mathrm{No}^{\mathrm{M}}$ & $\mathrm{Yes}^{\mathrm{M}}$ & $\mathrm{x}$ & $\mathrm{x}$ & $\mathrm{x}$ \\
\hline Barnes and Vidgen (2009) & $\mathrm{X}$ & $\mathrm{x}$ & Yes & $\mathrm{x}$ & $\mathrm{x}$ & Yes \\
\hline \multicolumn{7}{|l|}{ Brown et al. $(2010)$} \\
\hline Study 1 - Without Moderating Variable & No & No & No & $\mathrm{x}$ & Yes & Yes \\
\hline Study 1 - With Moderating Variable & $\mathrm{Yes}^{\mathrm{AG}}$ & Yes $^{\text {AGE }}$ & Yes $^{\text {AGE }}$ & Yes $^{\mathrm{AE}}$ & $\mathrm{Yes}^{\mathrm{AE}}$ & $\mathrm{x}$ \\
\hline Study 2 - Without Moderating Variable & No & No & No & $\mathrm{x}$ & No & Yes \\
\hline Study 2 - With Moderating Variable & $\mathrm{Yes}^{\mathrm{AG}}$ & Yes $^{\text {AGE }}$ & Yes $^{\text {AGE }}$ & $\mathrm{x}$ & $\mathrm{Yes}^{\mathrm{AE}}$ & $\mathrm{x}$ \\
\hline Butler and Richardson (2005) & Yes & Yes & $\mathrm{x}$ & $\mathrm{x}$ & $\mathrm{x}$ & $\mathrm{x}$ \\
\hline Carlsson et al. (2006) & Yes & Yes & Yes & No & $\mathrm{x}$ & Yes \\
\hline Carter and Schaupp (2009) & Yes & No & Yes & Yes & $\mathrm{x}$ & $\mathrm{x}$ \\
\hline Chang et al. (2007) & Yes & Yes & Yes & $\mathrm{x}$ & Yes & Yes \\
\hline Chen et al. (2008) & Yes & Yes & Yes & $\mathrm{x}$ & $\mathrm{x}$ & Yes \\
\hline Cheng et al. (2008a) & Yes & No & Yes & $\mathrm{x}$ & $\mathrm{x}$ & $\mathrm{x}$ \\
\hline Cheng et al. (2008b) & Yes & No & Yes & $\mathrm{x}$ & $\mathrm{x}$ & $\mathrm{x}$ \\
\hline Cheng et al. (2008c) & Yes & No & Yes & $\mathrm{X}$ & $\mathrm{x}$ & $\mathrm{X}$ \\
\hline Chiu et al. (2010) & Yes & No & Yes & Yes & $\mathrm{x}$ & Yes \\
\hline Chiu and Wang (2008) & Yes & Yes & No & No & $\mathrm{x}$ & $\mathrm{x}$ \\
\hline Cornacchia et al. (2008) & $\mathrm{x}$ & $\mathrm{X}$ & Yes & $\mathrm{x}$ & Yes & $\mathrm{x}$ \\
\hline Dasgupta and Gupta (2010) & $\mathrm{Yes}^{\mathrm{G}}$ & $\mathrm{Yes}^{\mathrm{G}}$ & $\mathrm{Yes}^{\mathrm{G}}$ & $\mathrm{x}$ & Yes & No \\
\hline \multicolumn{7}{|l|}{ Dulle and Minishi-Majanja (2011) } \\
\hline Model 1 & Yes & Yes & No & $\mathrm{x}$ & $\mathrm{x}$ & $\mathrm{x}$ \\
\hline Model 2 & $\mathrm{x}$ & $\mathrm{x}$ & $\mathrm{x}$ & $\mathrm{x}$ & Yes & $\mathrm{x}$ \\
\hline \multicolumn{7}{|l|}{ Duyck et al. (2008) } \\
\hline UTAUT & Yes & Yes & No & Yes & $\mathrm{X}$ & $\mathrm{X}$ \\
\hline UTAUT+Attitude & No & No & No & Yes & $\mathrm{x}$ & $\mathrm{x}$ \\
\hline UTAUT+Self-Efficacy & Yes & No & No & Yes & $\mathrm{x}$ & $\mathrm{x}$ \\
\hline UTAUT+Anxiety & Yes & No & No & Yes & $\mathrm{x}$ & $\mathrm{x}$ \\
\hline \multicolumn{7}{|l|}{ Duyck et al. (2010) } \\
\hline T1-Pre-Implementation Model & Yes & Yes & No & Yes & $\mathrm{x}$ & $\mathrm{x}$ \\
\hline T2-After 1-Year & Yes & No & Yes & Yes & Yes & No \\
\hline Pooled & Yes & Yes & Yes & Yes & $\mathrm{x}$ & $\mathrm{x}$ \\
\hline Foon and Fah (2011) & Yes & Yes & Yes & Yes & $\mathrm{x}$ & $\mathrm{x}$ \\
\hline Gupta et al. (2008) & $\mathrm{Yes}^{\mathrm{G}}$ & $\mathrm{Yes}^{\mathrm{G}}$ & $\mathrm{Yes}^{\mathrm{G}}$ & $\mathrm{x}$ & Yes & No \\
\hline
\end{tabular}




\begin{tabular}{|c|c|c|c|c|c|c|}
\hline \multicolumn{7}{|l|}{$\mathrm{He}$ and $\mathrm{Lu}(2007 \mathrm{a})$} \\
\hline Model 1 & Yes & No & Yes & $\mathrm{x}$ & Yes & Yes \\
\hline Model 2 & No & No & Yes & $\mathrm{x}$ & Yes & Yes \\
\hline Model 3 & No & No & Yes & $\mathrm{x}$ & Yes & Yes \\
\hline He and Wei (2009) & $\mathrm{x}$ & $\mathrm{x}$ & $\mathrm{x}$ & $\mathrm{x}$ & Yes & Yes \\
\hline Hung et al. (2007) & Yes & Yes & Yes & $\mathrm{x}$ & Yes & Yes \\
\hline Hutchison and Bekkering (2009) & No & No & No & No & $\mathrm{x}$ & $\mathrm{x}$ \\
\hline Jayasingh and Eze (2009) & $\mathrm{x}$ & $\mathrm{x}$ & Yes & $\mathrm{x}$ & $\mathrm{x}$ & $\mathrm{x}$ \\
\hline Johnston and Warkentin (2010) & $\mathrm{x}$ & $\mathrm{x}$ & Yes & $\mathrm{x}$ & $\mathrm{x}$ & $\mathrm{x}$ \\
\hline Jong and Wang (2009) & Yes & $\mathrm{x}$ & Yes & Yes & $\mathrm{x}$ & Yes \\
\hline Kijsanayotin et al. (2009) & Yes & Yes & Yes & $\mathrm{x}$ & Yes & Yes \\
\hline \multicolumn{7}{|l|}{ Koh et al. (2010) } \\
\hline Model 1 & Yes & $\mathrm{x}$ & Yes & $\mathrm{x}$ & $\mathrm{x}$ & No \\
\hline Model-2 & Yes & $\mathrm{x}$ & Yes & $\mathrm{x}$ & $\mathrm{x}$ & No \\
\hline Kourouthanassis et al. (2010) & Yes & No & Yes & $\mathrm{x}$ & $\mathrm{x}$ & $\mathrm{x}$ \\
\hline \multicolumn{7}{|l|}{ Laumer et al. (2010) } \\
\hline Under-Age Applicants & Yes & No & No & Yes & $\mathrm{x}$ & $\mathrm{x}$ \\
\hline Full-Age Applicants & Yes & No & Yes & No & $\mathrm{x}$ & $\mathrm{x}$ \\
\hline Lee and Lin (2008) & Yes & Yes & Yes & Yes & $\mathrm{x}$ & $\mathrm{x}$ \\
\hline Lee et al. (2010a) & Yes & No & Yes & $\mathrm{x}$ & Yes & Yes \\
\hline \multicolumn{7}{|l|}{ Lin et al. (2004) } \\
\hline Without Moderating Effect & No & Yes & $\mathrm{x}$ & No & $\mathrm{x}$ & Yes \\
\hline With Moderating Effect & $\mathrm{No}^{\mathrm{G}}$ & $\mathrm{No}^{\mathrm{GE}}$ & $\mathrm{x}$ & $\mathrm{x}$ & $\mathrm{No}^{\mathrm{E}}$ & Yes \\
\hline Liu et al. (2008) & Yes & No & Yes & $\mathrm{x}$ & $\mathrm{x}$ & $\mathrm{x}$ \\
\hline Louho et al. (2006) & Yes & Yes & No & No & $\mathrm{x}$ & No \\
\hline \multicolumn{7}{|l|}{ Luo et al. (2010) } \\
\hline PLS Analysis & Yes & $\mathrm{x}$ & $\mathrm{x}$ & $\mathrm{x}$ & $\mathrm{x}$ & $\mathrm{x}$ \\
\hline Post-Hoc Analysis & Yes & $\mathrm{x}$ & $\mathrm{x}$ & $\mathrm{x}$ & $\mathrm{x}$ & $\mathrm{x}$ \\
\hline \multicolumn{7}{|l|}{ Maldonado et al. (2009) } \\
\hline Without Moderating Effect & $\mathrm{x}$ & $\mathrm{x}$ & Yes & $\mathrm{x}$ & No & Yes \\
\hline With Moderating Effect on SI-->BI & $\mathrm{x}$ & $\mathrm{x}$ & Yes & $\mathrm{x}$ & No & Yes \\
\hline \multicolumn{7}{|l|}{ Maldonado et al. (2011) } \\
\hline Without Moderating Effect & $\mathrm{x}$ & $\mathrm{x}$ & Yes & $\mathrm{x}$ & No & Yes \\
\hline With Moderating Effect on SI-->BI & $\mathrm{x}$ & $\mathrm{x}$ & Yes & $\mathrm{x}$ & No & Yes \\
\hline Marchewka et al. (2007) & No & Yes & Yes & No & $\mathrm{x}$ & $\mathrm{x}$ \\
\hline Mayer et al. (2011) & Yes & Yes & Yes & $\mathrm{x}$ & $\mathrm{x}$ & $\mathrm{x}$ \\
\hline \multicolumn{7}{|l|}{ McLeod et al. (2009a) } \\
\hline Professionals & Yes & No & No & $\mathrm{x}$ & $\mathrm{x}$ & $\mathrm{x}$ \\
\hline Novices & No & Yes & Yes & $\mathrm{x}$ & $\mathrm{x}$ & $\mathrm{x}$ \\
\hline McLeod et al. (2009b) & Yes & Yes & Yes & $\mathrm{x}$ & $\mathrm{x}$ & $\mathrm{x}$ \\
\hline Neufeld et al. (2007) & Yes & Yes & Yes & $\mathrm{x}$ & Yes & Yes \\
\hline \multicolumn{7}{|l|}{ Niehaves and Plattfaut (2010) } \\
\hline Without Moderating Effect & Yes & Yes & Yes & $\mathrm{x}$ & Yes & Yes \\
\hline With Moderating Effect & No & No & No & $\mathrm{x}$ & No & Yes \\
\hline Nistor et al. (2010) & Yes & Yes & Yes & $\mathrm{x}$ & No & Yes \\
\hline Nov and Ye (2009) & Yes & Yes & $\mathrm{x}$ & $\mathrm{x}$ & $\mathrm{x}$ & $\mathrm{x}$ \\
\hline Or et al. (2011) & Yes & $\mathrm{x}$ & $\mathrm{x}$ & $\mathrm{x}$ & $\mathrm{x}$ & Yes \\
\hline Pahnila et al. (2011) & Yes & Yes & Yes & $\mathrm{x}$ & Yes & Yes \\
\hline Pai and Tu (2011) & No & Yes & Yes & $\mathrm{x}$ & Yes & Yes \\
\hline Pavon and Brown (2010) & Yes & No & $\mathrm{x}$ & $\mathrm{x}$ & $\mathrm{x}$ & $\mathrm{x}$ \\
\hline Payne (2008) & $\mathrm{No}^{\mathrm{AG}}$ & $\mathrm{No}^{\mathrm{AGE}}$ & $\mathrm{No}^{\mathrm{M}}$ & No & $\mathrm{Yes}^{\mathrm{AE}}$ & Yes \\
\hline \multicolumn{7}{|l|}{ Pynoo et al. (2008) } \\
\hline T1 - University Hospital & Yes & No & No & Yes & $\mathrm{x}$ & $\mathrm{x}$ \\
\hline T2 - University Hospital & Yes & No & No & No & No & Yes \\
\hline T3 - University Hospital & Yes & No & No & Yes & No & No \\
\hline T1 - Private Hospital & No & Yes & No & No & $\mathrm{x}$ & $\mathrm{x}$ \\
\hline T2 - Private Hospital & Yes & No & Yes & Yes & No & Yes \\
\hline
\end{tabular}




\begin{tabular}{|c|c|c|c|c|c|c|}
\hline T3 - Private Hospital & No & No & No & Yes & No & No \\
\hline \multicolumn{7}{|l|}{ Pynoo et al. (2011) } \\
\hline Time - T1 & Yes & No & Yes & No & $\mathrm{x}$ & $\mathrm{x}$ \\
\hline Time - T2 & Yes & No & No & No & $\mathrm{x}$ & $\mathrm{x}$ \\
\hline Time - T3 & No & Yes & Yes & No & $\mathrm{x}$ & $\mathrm{x}$ \\
\hline Pooled Over Three Measurements & Yes & No & Yes & No & $\mathrm{x}$ & $\mathrm{x}$ \\
\hline Sahu and Gupta (2007) & Yes & Yes & Yes & $\mathrm{x}$ & $\mathrm{x}$ & $\mathrm{x}$ \\
\hline Sambasivan et al. (2010) & $\mathrm{x}$ & $\mathrm{x}$ & $\mathrm{x}$ & Yes & $\mathrm{x}$ & $\mathrm{x}$ \\
\hline \multicolumn{7}{|l|}{ Sapio et al. (2010) } \\
\hline General STB Use & $\mathrm{x}$ & $\mathrm{x}$ & $\mathrm{x}$ & $\mathrm{x}$ & Yes & $\mathrm{x}$ \\
\hline Interactive Service Use & $\mathrm{x}$ & $\mathrm{x}$ & $\mathrm{x}$ & $\mathrm{x}$ & Yes & $\mathrm{x}$ \\
\hline Informative Service Use & $\mathrm{x}$ & $\mathrm{x}$ & $\mathrm{x}$ & $\mathrm{x}$ & No & $\mathrm{x}$ \\
\hline Schaupp et al. (2010) & Yes & No & Yes & Yes & $\mathrm{x}$ & $\mathrm{x}$ \\
\hline Shi (2009) & Yes & Yes & No & Yes & $\mathrm{x}$ & $\mathrm{x}$ \\
\hline \multicolumn{7}{|l|}{ Shin (2009) } \\
\hline Initial Model & $\mathrm{x}$ & $\mathrm{x}$ & No & $\mathrm{x}$ & $\mathrm{x}$ & Yes \\
\hline Extended Model & $\mathrm{x}$ & $\mathrm{x}$ & $\mathrm{Yes}^{\mathrm{B}}$ & $\mathrm{x}$ & $\mathrm{x}$ & Yes \\
\hline Shin (2010) & $\mathrm{x}$ & $\mathrm{x}$ & Yes & $\mathrm{x}$ & $\mathrm{x}$ & Yes \\
\hline Song and Han (2009) & Yes & Yes & $\mathrm{x}$ & $\mathrm{x}$ & $\mathrm{x}$ & $\mathrm{x}$ \\
\hline Sumak et al. (2010) & No & No & Yes & $\mathrm{x}$ & Yes & Yes \\
\hline Tan and Wu (2010) & $\mathrm{x}$ & $\mathrm{x}$ & Yes & $\mathrm{x}$ & $\mathrm{x}$ & $\mathrm{x}$ \\
\hline Teo (2011) & $\mathrm{x}$ & $\mathrm{x}$ & $\mathrm{x}$ & Yes & $\mathrm{x}$ & $\mathrm{x}$ \\
\hline Tibenderana et al. (2010) & No & $\mathrm{x}$ & Yes & $\mathrm{x}$ & Yes & $\mathrm{x}$ \\
\hline \multicolumn{7}{|l|}{ Tsai et al. (2009) } \\
\hline Model 1 & $\mathrm{x}$ & $\mathrm{x}$ & Yes & Yes & $\mathrm{x}$ & $\mathrm{x}$ \\
\hline Model 2 & $\mathrm{x}$ & $\mathrm{x}$ & Yes & Yes & $\mathrm{x}$ & $\mathrm{x}$ \\
\hline Model 3 & $\mathrm{x}$ & $\mathrm{x}$ & Yes & Yes & $\mathrm{x}$ & $\mathrm{x}$ \\
\hline Udeh (2008) & $\mathrm{x}$ & $\mathrm{x}$ & $\mathrm{x}$ & $\mathrm{x}$ & Yes & $\mathrm{x}$ \\
\hline van Biljon and Kotze (2008) & $\mathrm{x}$ & $\mathrm{x}$ & Yes & Yes & $\mathrm{x}$ & $\mathrm{x}$ \\
\hline van Dijk et al. (2008) & Yes & Yes & $\mathrm{x}$ & $\mathrm{x}$ & $\mathrm{x}$ & $\mathrm{x}$ \\
\hline \multicolumn{7}{|l|}{ van Schaik (2009) } \\
\hline Study 1 - Virtual Learning Environment & No & No & Yes & $\mathrm{x}$ & No & No \\
\hline Study 1 - Library Website & Yes & Yes & No & $\mathrm{x}$ & No & Yes \\
\hline Study 2 - Site 1 - Library Website & Yes & Yes & No & $\mathrm{x}$ & Yes & No \\
\hline Study 2 - Site 2 - Goal Mode & No & No & Yes & $\mathrm{x}$ & $\mathrm{x}$ & Yes \\
\hline Study 2 - Site 3 - Action Mode & Yes & Yes & No & $\mathrm{x}$ & $\mathrm{x}$ & Yes \\
\hline Venkatesh et al. (2008) & $\mathrm{x}$ & $\mathrm{x}$ & $\mathrm{x}$ & $\mathrm{x}$ & Yes & Yes \\
\hline \multicolumn{7}{|l|}{ Wang and Shih (2009) } \\
\hline Younger People & Yes & Yes & Yes & $\mathrm{x}$ & Yes & Yes \\
\hline Older People & Yes & Yes & Yes & $\mathrm{x}$ & Yes & Reverse \\
\hline All Respondents & Yes & Yes & Yes & $\mathrm{x}$ & Yes & Yes \\
\hline \multicolumn{7}{|l|}{ Wang et al. (2009) } \\
\hline For Males & Yes & Yes & Yes & $\mathrm{x}$ & $\mathrm{x}$ & $\mathrm{x}$ \\
\hline For Females & Yes & Yes & No & $\mathrm{x}$ & $\mathrm{x}$ & $\mathrm{x}$ \\
\hline For Younger People & Yes & $\mathrm{x}$ & $\mathrm{x}$ & $\mathrm{x}$ & $\mathrm{x}$ & $\mathrm{x}$ \\
\hline For Older People & Yes & Yes & Yes & $\mathrm{x}$ & $\mathrm{x}$ & $\mathrm{x}$ \\
\hline For All Respondents & Yes & Yes & Yes & $\mathrm{x}$ & $\mathrm{x}$ & $\mathrm{x}$ \\
\hline Weerakkody et al. (2009) & Yes & Yes & Yes & Reverse & $\mathrm{x}$ & Yes \\
\hline Wang et al. (2010) & Yes & Yes & Yes & $\mathrm{x}$ & $\mathrm{x}$ & $\mathrm{x}$ \\
\hline Wills et al. (2008) & Yes & Yes & Yes & $\mathrm{x}$ & Yes & Yes \\
\hline Wu et al. (2007) & Yes & No & Yes & Yes & Yes & Yes \\
\hline Wu et al. (2008) & Yes & No & Yes & Yes & Yes & Yes \\
\hline \multicolumn{7}{|l|}{ Xu and Gupta (2009) } \\
\hline \begin{tabular}{|l|} 
Potential Users \\
\end{tabular} & Yes & Yes & $\mathrm{x}$ & $\mathrm{x}$ & $\mathrm{x}$ & $\mathrm{x}$ \\
\hline Experienced Users & Yes & Yes & $\mathrm{x}$ & $\mathrm{x}$ & $\mathrm{x}$ & $\mathrm{x}$ \\
\hline Yang (2010) & Yes & No & Yes & Yes & $\mathrm{x}$ & $\mathrm{x}$ \\
\hline Yao and Murphy (2007) & & & & & & \\
\hline
\end{tabular}




\begin{tabular}{|l|c|c|c|c|c|c|}
\hline For Women & $\mathrm{x}$ & Yes & $\mathrm{x}$ & $\mathrm{x}$ & $\mathrm{x}$ & $\mathrm{x}$ \\
\hline For Men & $\mathrm{x}$ & No & $\mathrm{x}$ & $\mathrm{x}$ & $\mathrm{x}$ & $\mathrm{x}$ \\
\hline For Overall Voters & $\mathrm{x}$ & Yes & $\mathrm{x}$ & $\mathrm{x}$ & $\mathrm{x}$ & $\mathrm{x}$ \\
\hline Yeow and Loo (2009) & \multicolumn{7}{|l|}{} \\
\hline MyKad Touch n' Go Application & Yes & Yes & Yes & Yes & $\mathrm{x}$ & $\mathrm{x}$ \\
\hline MyKad ATM Application & Yes & Yes & Yes & No & $\mathrm{x}$ & $\mathrm{x}$ \\
\hline Zhang et al. (2010) & Yes & No & Yes & $\mathrm{x}$ & $\mathrm{x}$ & $\mathrm{x}$ \\
\hline Without Moderating Variable & No & No & No & $\mathrm{x}$ & $\mathrm{x}$ & $\mathrm{x}$ \\
\hline With Moderating Variable (Gender) & Yes & No & Yes & Yes & $\mathrm{x}$ & Yes \\
\hline Zhou (2008) &
\end{tabular}

[Legend: Yes: Relation was found to be significant and positive, No: Relation was found to be non-significant, $\mathbf{x}$ : Relation was not examined, Reverse: Relation was found to be significant but negative, $\mathbf{Y e s}^{\mathbf{M}} / \mathbf{N o}^{\mathbf{M}}$ : Relation was significant/non-significant due to moderating effect of all moderating variables age, gender, experience, and voluntariness of use, $\mathbf{Y e s}^{\mathbf{G}} / \mathbf{N o}^{\mathbf{G}}$ : Relation was significant/non-significant due to moderating variable gender,

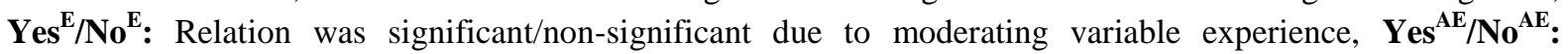
Relation was significant/non-significant due to moderating variable age and experience, $\mathbf{Y e s}^{\mathbf{A G}} / \mathbf{N o}^{\mathbf{A G}}$ : Relation was significant/non-significant due to moderating variable age and gender, $\mathbf{Y e s}^{\mathbf{A G E}} / \mathbf{N o}^{\mathbf{A G E}}$ : Relation was significant/non-significant due to moderating variable age, gender, and experience, $\mathbf{Y e s}^{\mathbf{B}}$ : Relationship of SI was found to be significant both on BI and Usage]

\subsubsection{Weight Analysis}

In order to better understand the predictive power of each individual independent variable, a weight analysis was performed for each independent/dependent variable pairing. We adopted an approach in line with the work of Jeyaraj et al. (2006) in order to identify the most/least frequently used predictors, and among these, the best, worst, and promising predictors. Data for this analysis was extracted from Table 11 (and is summarised in Table 12), weights being calculated by the value obtained by dividing the number of times a particular independent/dependent variable relationship was found to be significant by the total number of times that the relationship had been examined across all studies.

Table 12

Relationships between Major UTAUT Variables

\begin{tabular}{|l|c|c|c|c|c|c|}
\hline Relations & PE-BI & EE-BI & SI-BI & FC-BI & FC-U & BI-U \\
\hline Significant Relation & 93 & 64 & 86 & 32 & 36 & 49 \\
\hline Non-Significant Relation & 23 & 46 & 29 & 15 & 18 & 11 \\
\hline Negative Relation & 0 & 0 & 0 & 1 & 0 & 1 \\
\hline Not Tested & 33 & 39 & 34 & 101 & 95 & 88 \\
\hline Total & 149 & 149 & 149 & 149 & 149 & 149 \\
\hline Total Relations Examined & 116 & 110 & 115 & 48 & 54 & 61 \\
\hline Total \# of Significant Relations & 93 & 64 & 86 & 33 & 36 & 50 \\
\hline \multirow{2}{*}{ Weight of Predictors } & $93 / 116$ & $64 / 110$ & $86 / 115$ & $33 / 48$ & $36 / 54$ & $50 / 61$ \\
\cline { 2 - 7 } & $=0.80$ & $=0.58$ & $=0.75$ & $=0.69$ & $=0.67$ & $=0.82$ \\
\hline
\end{tabular}

A weight of ' 1 ' indicates that the relationship between the two constructs is significant across all studies, whereas ' 0 ' indicates that this relationship is non-significant across all the studies examined. The weights are an indication of the analytical power of an independent variable. However, care must be taken whilst considering these values, as it is not simply a weight of ' 1 ' that would declare a variable as being a best predictor. It is also important to note how many times a particular relationship was examined, as consistent evidence across studies is required in order that a best predictor be identified (Jeyaraj et al., 2006).
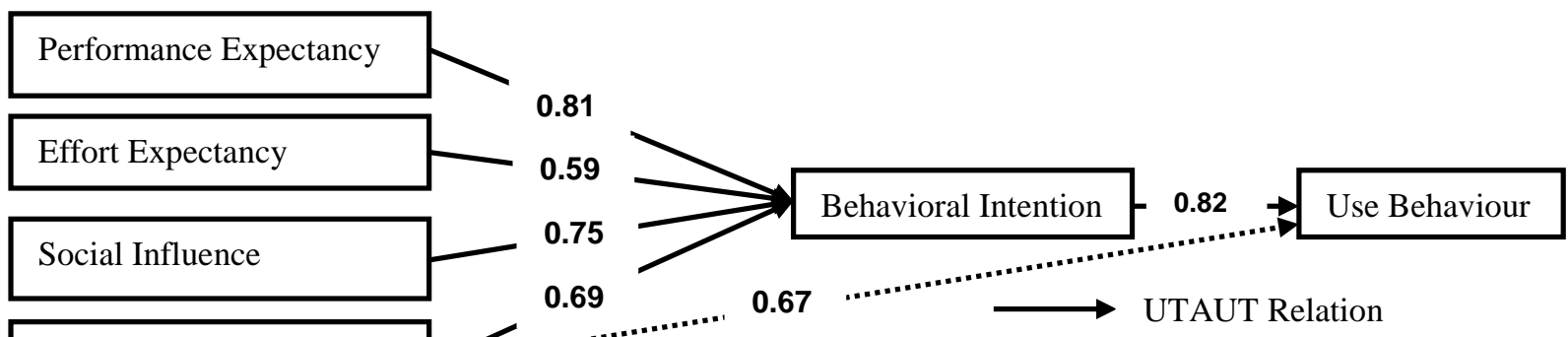
Fig. 2. Weight Significance between UTAUT Constructs (Source: Venkatesh et al., 2003)

This was not particular an issue in our study, as all relationships had been examined numerous times. Jeyaraj et al. (2006) suggest that a weight of 0.80 or more is required for an independent variable to qualify as a best predictor, and we adopt this threshold in our work. Figure 2 illustrates the predictive power of the independent variables of UTAUT. Weight analysis of the independent variables indicates that only two variables (PE and $\mathrm{BI}$ ) qualify for the best predictor category, whereas the other variables did not meet this requirement, the closest being social influence, with a weight of 0.74 .

\subsection{Analysis of External Variables}

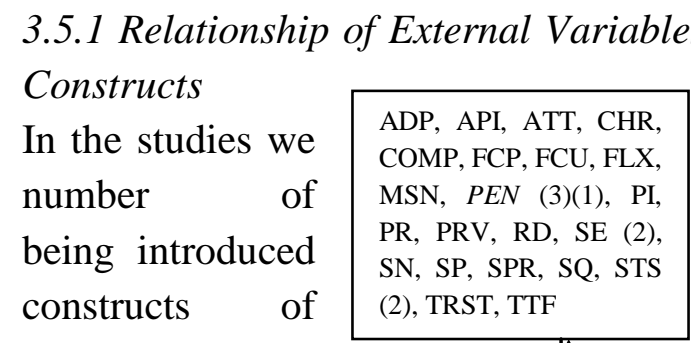

AC, AG (2), ANX (5), ASS, ATT (11), AV, AWR, CA, CAX (2), CB, CO, COMP (2), CSE (2), CWE, DMA, DME, DMP, EDU, ES, FP, GEN (2), IQ, IV, KS, LC, MB, MT (2), OB (2), OE, ON, PC (3), PCM, PCS, PI (3), PEOU (8), PIN, POC, PP (2), PQ, PR (3), PRV (2), PS, PSC (2), PT, PU (8), RA, RE, REL, SA, SB, SBL, SC, SE (8), SML, SN (3), SP, STS (4), TEF, TI (2), TL, TMS, TOI, TRN (2), TRST (5), TTF, UV, VOL (2), WDSE

examined,

external variables onto the major UTAUT. In keeping with the of Lee et al. (2003) their work on

TAM, we illustrate
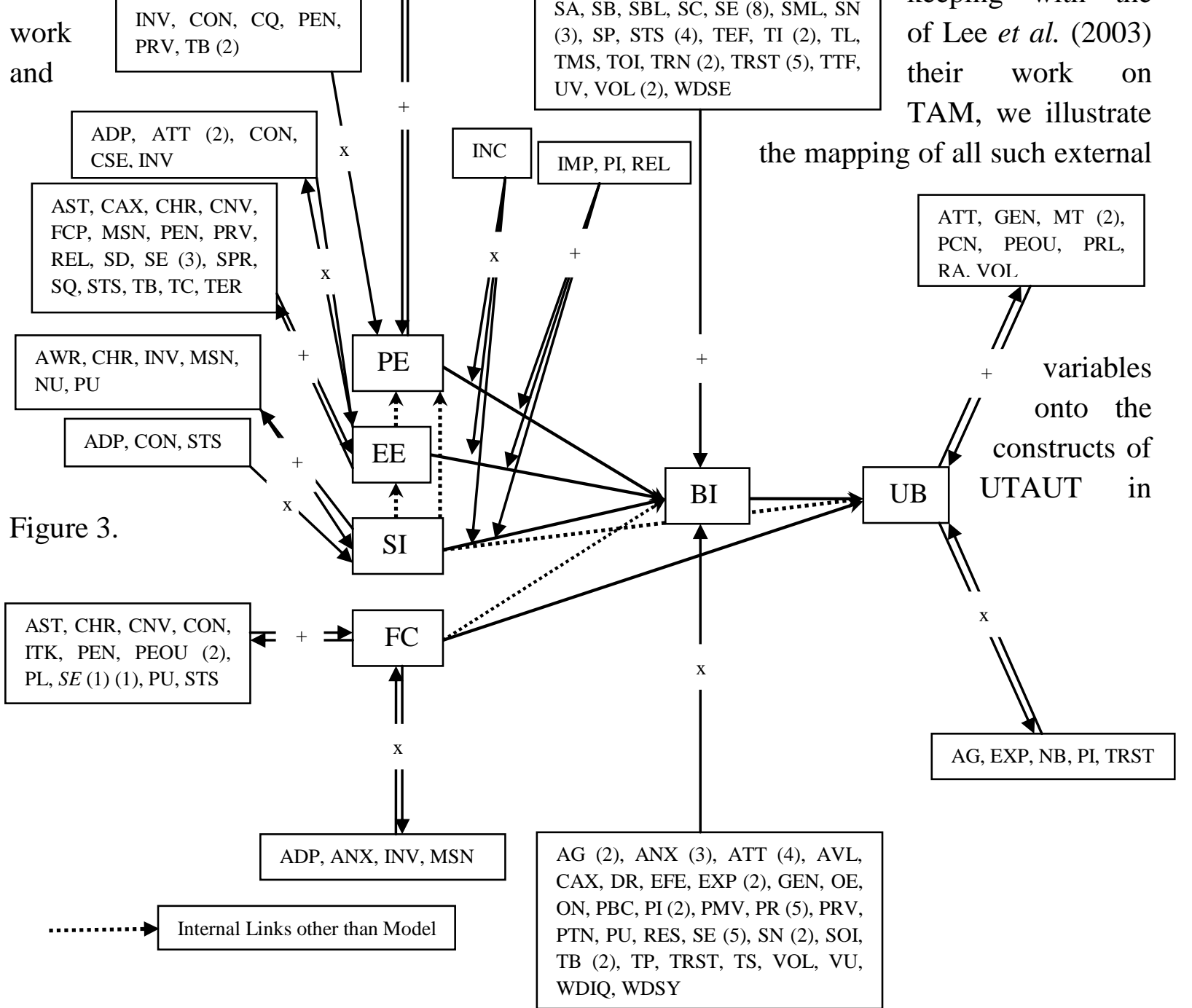
Fig. 3. Diagrammatic Representation of External Variables (Approach Adapted from Lee et al., 2003)

\subsubsection{Most Frequently Used External Variables}

Table 13 presents the 20 most frequently used external variables that affect PE, EE, SI, FC, $\mathrm{BI}$, or $\mathrm{UB}$, and provides the definitions as given in their originating studies. Results reveal that self-efficacy ( 21 occurrences) is most often used external variable, closely followed by attitude (20 occurrences) and trust (18 occurrences). Comparing these results with those of Lee et al. (2003) in their study of TAM reveals that self-efficacy, personal innovativeness, subjective norms, voluntariness, computer anxiety, compatibility, and relative advantage were examined a number of times across both TAM and UTAUT studies.

Table 13

Variables Used in UTAUT Research (Approach from Lee et al., 2003)

\begin{tabular}{|c|c|c|c|}
\hline EV (\#) & Definition & Origin & Referred Articles \\
\hline SE (21) & $\begin{array}{l}\text { The belief that one has the capability to } \\
\text { perform a particular behavior }\end{array}$ & Bandura (1977) & $\begin{array}{l}\text { Hutchison and Bekkering } \\
(2009), \text { Johnston and Warkentin } \\
(2010)\end{array}$ \\
\hline $\operatorname{ATT}(20)$ & Person's evaluation of a specified behavior & $\begin{array}{l}\text { Fishbein and Ajzen } \\
(1975)\end{array}$ & $\begin{array}{l}\text { Al-Somali et al. (2009), } \\
\text { Hutchison and Bekkering (2009) }\end{array}$ \\
\hline TRST (18) & $\begin{array}{l}\text { Willingness of party to be vulnerable to the } \\
\text { actions of the another party based on the } \\
\text { expectations that the other party will } \\
\text { perform a particular action important to the } \\
\text { trustor }\end{array}$ & Mayers et al. (1995) & $\begin{array}{l}\text { Foon and Fah (2011), } \\
\text { Sambasivan et al. (2010) }\end{array}$ \\
\hline PU (15) & $\begin{array}{l}\text { The user's perception to the extent that the } \\
\text { system will improve the user's workplace } \\
\text { performance-- }\end{array}$ & Davis (1989) & $\begin{array}{l}\text { Ambali (2009), Barnes and } \\
\text { Vidgen (2009) }\end{array}$ \\
\hline
\end{tabular}




\begin{tabular}{|c|c|c|c|}
\hline PEOU (15) & $\begin{array}{l}\text { The extent to which a user believes that } \\
\text { using a particular system will be effortless }\end{array}$ & Davis (1989) & Shin (2010), Udeh (2008) \\
\hline ANX (12) & $\begin{array}{l}\text { An unpleasant emotional state or condition } \\
\text { which is characterized by subjective } \\
\text { feelings of tension, apprehension, and } \\
\text { worry }\end{array}$ & Spielberger (1972) & $\begin{array}{l}\text { AbuShanab et al. (2010), } \\
\text { Carlsson et al. (2006) }\end{array}$ \\
\hline PR (10) & $\begin{array}{l}\text { A combination of uncertainty and plus } \\
\text { seriousness of outcome involved }\end{array}$ & Bauer (1960) & $\begin{array}{l}\text { Abu-Shanab and Pearson } \\
(2009) \text {, Luo et al. }(2010)\end{array}$ \\
\hline PI (8) & $\begin{array}{l}\text { An individual trait reflecting a willingness } \\
\text { to try out any new technology }\end{array}$ & $\begin{array}{l}\text { Agarwal and } \\
\text { Karahanna (2000) }\end{array}$ & $\begin{array}{l}\text { Jayasingh and Eze (2009), Xu } \\
\text { and Gupta (2009) }\end{array}$ \\
\hline STS (7) & $\begin{array}{l}\text { The attitude that a user has toward an } \\
\text { information system }\end{array}$ & $\begin{array}{l}\text { DeLone and } \\
\text { McLean (1992) }\end{array}$ & $\begin{array}{l}\text { Chan et al. (2010), Liu et al. } \\
(2008)\end{array}$ \\
\hline TB (7) & $\begin{array}{l}\text { The perception that the trustworthiness of } \\
\text { the vendor consists of a set of specific } \\
\text { beliefs about integrity, benevolence, and } \\
\text { competence }\end{array}$ & $\begin{array}{l}\text { McKnight and } \\
\text { Chervany (2002) }\end{array}$ & Luo et al. (2010), Shi (2009) \\
\hline $\mathbf{S N}(6)$ & $\begin{array}{l}\text { Person's perception that most people who } \\
\text { are important to him think he should or } \\
\text { should not perform the behavior in question }\end{array}$ & $\begin{array}{l}\text { Fishbein and Ajzen } \\
(1975)\end{array}$ & $\begin{array}{l}\text { Laumer et al. (2010), Or et al. } \\
(2010)\end{array}$ \\
\hline VOL (6) & $\begin{array}{l}\text { The degree to which use of the innovation is } \\
\text { perceived as being voluntary }\end{array}$ & $\begin{array}{l}\text { Moore and } \\
\text { Benbasat (1991) }\end{array}$ & \begin{tabular}{|l} 
Anderson et al. (2006), \\
Hutchison and Bekkering (2009)
\end{tabular} \\
\hline CAX (5) & $\begin{array}{l}\text { An individual's apprehension, or even fear, } \\
\text { when she/he is faced with the possibility of } \\
\text { using computers }\end{array}$ & $\begin{array}{l}\text { Simonson et al. } \\
(1987)\end{array}$ & $\begin{array}{l}\text { Lin et al. (2004), Nistor et al. } \\
(2010)\end{array}$ \\
\hline CSE (5) & $\begin{array}{l}\text { An individual judgment of one's capability } \\
\text { to use a computer }\end{array}$ & $\begin{array}{ll}\text { au } & \text { and } \\
\text { s (1995) } & \end{array}$ & $\begin{array}{l}\text { Chiu and Wang (2008), Nov and } \\
\text { Ye (2009) }\end{array}$ \\
\hline PEN (4) & $\begin{array}{l}\text { The extent to which the activity of using a } \\
\text { specific system is perceived to be enjoyable } \\
\text { in its own right, aside from any } \\
\text { performance consequences resulting from } \\
\text { system usage }\end{array}$ & Davis et al. (1992) & $\begin{array}{l}\text { Song and Han (2009), Wu et al. } \\
(2010)\end{array}$ \\
\hline COMP (3) & $\begin{array}{l}\text { The degree to which an innovation is } \\
\text { perceived as being consistent with the } \\
\text { existing values, needs, and past experiences } \\
\text { of potential adopters }\end{array}$ & Rogers (1983) & $\begin{array}{l}\text { Chan et al. (2010), Jayasingh } \\
\text { and Eze (2009) }\end{array}$ \\
\hline PC (3) & $\begin{array}{l}\text { The belief that the promise of another can } \\
\text { be relied upon even under unforeseen } \\
\text { circumstances }\end{array}$ & Suh and Han (2002) & $\begin{array}{l}\text { YenYuen and Yeow (2009), } \\
\text { Yeow and Loo (2009) }\end{array}$ \\
\hline $\mathrm{RD}(2)$ & $\begin{array}{l}\text { The degree to which the results of } \\
\text { adopting/using the IS innovation are } \\
\text { observable and communicable to others }\end{array}$ & Rogers (1983) & $\begin{array}{l}\text { Keller et al. (2007), Nov and Ye } \\
(2009)\end{array}$ \\
\hline $\mathbf{R A}(2)$ & $\begin{array}{l}\text { The degree to which an innovation is } \\
\text { perceived as being better than its precursor }\end{array}$ & Rogers (1983) & Shin (2010), Udeh (2008) \\
\hline OB (2) & $\begin{array}{l}\text { A systematic error in perception of an } \\
\text { individual's own standing relative to group } \\
\text { averages, in which negative events are seen } \\
\text { as less likely to occur to the individual than } \\
\text { average compared with the group, and } \\
\text { positive events as more likely to occur than } \\
\text { average compared with the group }\end{array}$ & Weinstein (1980) & $\begin{array}{l}\text { Carter and Schaupp } \\
\text { Schaupp et al. (2010) }\end{array}$ \\
\hline
\end{tabular}

[Legend: ANX: Anxiety, ATT: Attitude, CAX: Computer Anxiety, COMP: Compatibility, CSE: Computer Self-Efficacy, EV (\#): External Variable with its Occurrences across Relevant Studies; OB: Optimism Bias, PC: Perceived Credibility, PEN: Perceived Enjoyment, PEOU: Perceived Ease of Use, PI: Perceived Innovativeness, PR: Perceived Risk, PU: Perceived Usefulness, RA: Relative Advantage, RD: Result Demonstrability, SE: Self-Efficacy, SN: Subjective Norms, STS: Satisfaction, TB: Trust Belief, TRST: Trust, VOL: Voluntariness, Bold: Indicates the external variables were used across both studies of TAM (by Legris et al., 2003) and UTAUT analysed here] 


\subsection{Major Limitations of UTAUT Studies}

An analysis of acknowledged limitations across studies indicated that focusing on a single subject - in terms of a community, culture, country, organisation, agency, department, person, or age group - was the most widespread reported constraint (35 studies). This was followed by 27 studies acknowledging their focus on a single task at a given point of time, and hence according to Lee et al. (2003), limiting the potential of generalization of findings - a key weakness. In Lee et al's (2003) work on TAM, self-reported usage was the weakness most often acknowledged, whereas in our study, it appeared in seventh place in our list of acknowledged limitations. A series of additional limitations (including limited sample size, use of students to explore workplace issues, no use of moderating variables, and lack of exogenous factors) were also reported in the literature. Details of these and others are presented in Table 14, along with associated references. Nine limitations were reported only in a single study - these are documented in Table 14 in the other limitations category.

Table 14

Limitations in UTAUT Studies (Approach from Lee et al., 2003)

\begin{tabular}{|c|c|c|c|}
\hline Limitation & \# & Explanation & Studies \\
\hline $\begin{array}{l}\text { Single Subject/ } \\
\text { Biased Sample }\end{array}$ & 35 & $\begin{array}{l}\text { Sample based on only one or } \\
\text { limited community, culture, } \\
\text { country, organisation, agency, } \\
\text { department, person, or age- } \\
\text { group }\end{array}$ & $\begin{array}{l}\text { Aggelidis and Chatzoglou (2009), Brown and } \\
\text { Venkatesh (2005), Brown et al. (2010), Chang et al. } \\
\text { (2007), Chiu et al. (2010), Dasgupta and Gupta (2010), } \\
\text { He and Lu (2007a), Ho and Chou (2009), Hung et al. } \\
\text { (2007), Jayasingh and Eze (2009), Koh et al. (2010), } \\
\text { Koivumaki et al. (2008), Laumer et al. (2010), Lee et } \\
\text { al. (2010a), Li (2010), Mahzan and Lymer (2008), } \\
\text { Maldonado et al. (2009), Maldonado et al. (2011), } \\
\text { Niehaves and Plattfaut (2010), Or et al. (2011), Pai and } \\
\text { Tu (2011), Sahu and Gupta (2007), Samoutis et al. } \\
\text { (2008), Schaper and Pervan (2006), Shin (2009), } \\
\text { Tibenderana et al. (2010), Tsai et al. (2009), Wang and } \\
\text { Shih (2009), Wang et al. (2009), Wu et al. (2007), } \\
\text { YenYuen and Yeow (2009), Yeow and Loo (2009), } \\
\text { Yeow et al. (2008), Yao and Murphy (2007), Zhou et } \\
\text { al. (2010) }\end{array}$ \\
\hline Single Task & 27 & $\begin{array}{l}\text { Difficult to generalize the } \\
\text { result }\end{array}$ & $\begin{array}{l}\text { Abu-Shanab and Pearson (2009), Aggelidis and } \\
\text { Chatzoglou (2009), Alapetite et al. (2009), AlAwadhi } \\
\text { and Morris (2011), Brown and Venkatesh (2005), } \\
\text { Carter and Schaupp (2009), Chang et al. (2007), Chiu } \\
\text { and Wang (2008), Hung et al. (2007), Huser et al. } \\
\text { (2010), Kijsanayotin et al. (2009), Mahzan and Lymer } \\
\text { (2008), Maldonado et al. (2009), Maldonado et al. } \\
\text { (2011), Mayer et al. (2011), Schaper and Pervan } \\
\text { (2006), Shin (2009), Shin (2010), Sumak et al. (2010), } \\
\text { Teo (2011), Terzis and Economides (2011), Tsai et al. } \\
\text { (2009), van Raaij and Schepers (2008), Wang and Shih } \\
\text { (2009), Xu and Gupta (2009), Yao and Murphy (2007), } \\
\text { Zhou et al. (2010) }\end{array}$ \\
\hline $\begin{array}{l}\text { Cross Sectional } \\
\text { Study }\end{array}$ & 17 & One time cross-sectional study & $\begin{array}{l}\text { Al-Gahtani et al. (2007), Aoun et al. (2010), Chiu et al. } \\
\text { (2010), Chiu and Wang (2008), Heikkila and Smale } \\
\text { (2010), Hung et al. (2007), Kijsanayotin et al. (2009), } \\
\text { Luo et al. (2010), Neufeld et al. (2007), Sambasivan et } \\
\text { al. (2010), Schaupp et al. (2010), Shin (2010), } \\
\text { Tibenderana et al. (2010), Wang et al. (2009), Wills et } \\
\text { al. (2008), Wu et al. (2007), Zhou et al. (2010) }\end{array}$ \\
\hline
\end{tabular}




\begin{tabular}{|c|c|c|c|}
\hline $\begin{array}{l}\text { Limited Sample } \\
\text { Size }\end{array}$ & 14 & Small sample size & $\begin{array}{l}\text { Aggelidis and Chatzoglou (2009), Chiu and Eysenbach } \\
\text { (2010), Duyck et al. (2008), Foon and Fah (2011), } \\
\text { Hutchison and Bekkering (2009), Lee et al. (2010a), } \\
\text { Maldonado et al. (2009), Marchewka et al. (2007), } \\
\text { McLeod et al. (2009a), Pynoo et al. (2011), Or et al. } \\
\text { (2011), Trimmer et al. (2008), van Raaij and Schepers } \\
\text { (2008), Wills et al. (2008) }\end{array}$ \\
\hline Single IS & 12 & $\begin{array}{l}\text { Only a Single IS for the } \\
\text { research }\end{array}$ & $\begin{array}{l}\text { Aggelidis and Chatzoglou (2009), Aoun et al. (2010), } \\
\text { Brown et al. (2010), Carlsson et al. (2006), Chang et al. } \\
\text { (2007), Chiu and Wang (2008), Gupta et al. (2008), Im } \\
\text { et al. (2008), Sambasivan et al. (2010), Wang and Shih } \\
\text { (2009), Wang et al. (2009), Zhou et al. (2010) }\end{array}$ \\
\hline $\begin{array}{l}\text { Limited external } \\
\text { factors or } \\
\text { variables }\end{array}$ & 11 & $\begin{array}{l}\text { Counted number of external } \\
\text { constructs }\end{array}$ & $\begin{array}{l}\text { Chan et al. (2010), Chiu and Eysenbach (2010), Gupta } \\
\text { et al. (2008), He and Lu (2008), He et al. (2007), Im et } \\
\text { al. (2008), Or et al. (2011), Schaupp et al. (2010), } \\
\text { Terzis and Economides (2011), van Schaik (2009), Wu } \\
\text { et al. (2007) }\end{array}$ \\
\hline $\begin{array}{l}\text { Self-reported } \\
\text { Usage }\end{array}$ & 11 & $\begin{array}{l}\text { Did not measure the actual } \\
\text { usage }\end{array}$ & $\begin{array}{l}\text { Abu-Shanab and Pearson (2007), Abu-Shanab and } \\
\text { Pearson (2009), de Silva and Ratnadiwakara (2009), } \\
\text { Hung et al. (2007), Jayasingh and Eze (2009), Lin et al. } \\
\text { (2004), Luo et al. (2010), van Schaik (2009), } \\
\text { Venkatesh et al. (2008), Wang et al. (2009), Zhang et } \\
\text { al. (2010) }\end{array}$ \\
\hline Student Sample & 11 & $\begin{array}{l}\text { Improper to reflect the real } \\
\text { working environment }\end{array}$ & $\begin{array}{l}\text { AlAwadhi and Morris (2011), Carter and Schaupp } \\
\text { (2009), Im et al. (2008), Johnston and Warkentin } \\
(2010) \text {, Luo et al. (2010), McLeod et al. (2009a), } \\
\text { McLeod et al. (2009b), Sumak et al. (2010), Tsai et al. } \\
\text { (2009), Wu et al. (2010), Zhang et al. (2010) }\end{array}$ \\
\hline $\begin{array}{l}\text { Self- } \\
\text { reported/selection } \\
\text { Bias }\end{array}$ & 7 & $\begin{array}{l}\text { Poll suffering from self- } \\
\text { selecting opinion }\end{array}$ & $\begin{array}{l}\text { Carter and Schaupp (2009), Chan et al. (2010), Chiu et } \\
\text { al. (2010), Chiu and Wang (2008), Sahu and Gupta } \\
\text { (2007), Schaupp et al. (2010), Teo (2011) }\end{array}$ \\
\hline $\begin{array}{l}\text { No moderating } \\
\text { variables }\end{array}$ & 6 & No moderating variables used & $\begin{array}{l}\text { Al-Sobhi et al. (2011), Chen et al. (2008), Huser et al. } \\
\text { (2010), Loo et al. (2009), Yeow and Loo (2009), } \\
\text { YenYuen and Yeow (2009) }\end{array}$ \\
\hline $\begin{array}{l}\text { Explanatory } \\
\text { Study/ Incomplete } \\
\text { research }\end{array}$ & 4 & Partially complete research & $\begin{array}{l}\text { Barati and Mohammadi (2009), Debuse et al. (2008), } \\
\text { Koivumaki et al. (2008), YenYuen and Yeow (2009) }\end{array}$ \\
\hline $\begin{array}{l}\text { Limited exposure } \\
\text { to the technology }\end{array}$ & 3 & $\begin{array}{l}\text { Target population least aware } \\
\text { to technology }\end{array}$ & $\begin{array}{l}\text { Huser et al. (2010), Hutchison and Bekkering (2009), } \\
\text { Shin (2009) }\end{array}$ \\
\hline $\begin{array}{l}\text { Common method } \\
\text { bias/variance }\end{array}$ & 3 & $\begin{array}{|lll|}\begin{array}{l}\text { Data collected using same } \\
\text { survey }\end{array} & \\
\end{array}$ & $\begin{array}{l}\text { Laumer et al. (2010), Tsai et al. (2009), Wang et al. } \\
(2009)\end{array}$ \\
\hline \begin{tabular}{|l|} 
Gender Bias - \\
Majority \\
female/male \\
\end{tabular} & 2 & $\begin{array}{l}\text { Majority of either male or } \\
\text { female }\end{array}$ & Sumak et al. (2010), Wills et al. (2008) \\
\hline Male sample & 2 & Only male respondents & Al-Sobhi et al. (2011), Dasgupta and Gupta (2010) \\
\hline $\begin{array}{l}\text { Low R-Square/ } \\
\text { Unexplained } \sigma^{2} \\
\end{array}$ & 2 & Variance not explained & Hung et al. (2007), Teo (2011) \\
\hline Other Limitations & 9 & 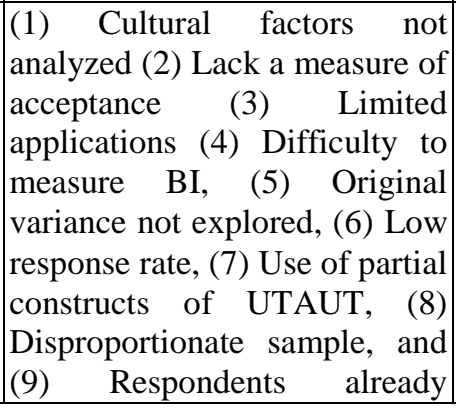 & $\begin{array}{l}\text { (1) de Silva and Ratnadiwakara (2009), (2) Duyck et } \\
\text { al. (2010), (3) Loo et al. (2009), (4) Neufeld et al. } \\
\text { (2007), (5) Pai and Tu (2011), (6) Pynoo et al. (2011), } \\
\text { (7) Shamsuddin (2009), (8) Shin (2009), and (9) } \\
\text { Sumak et al. (2010) } \\
\end{array}$ \\
\hline
\end{tabular}




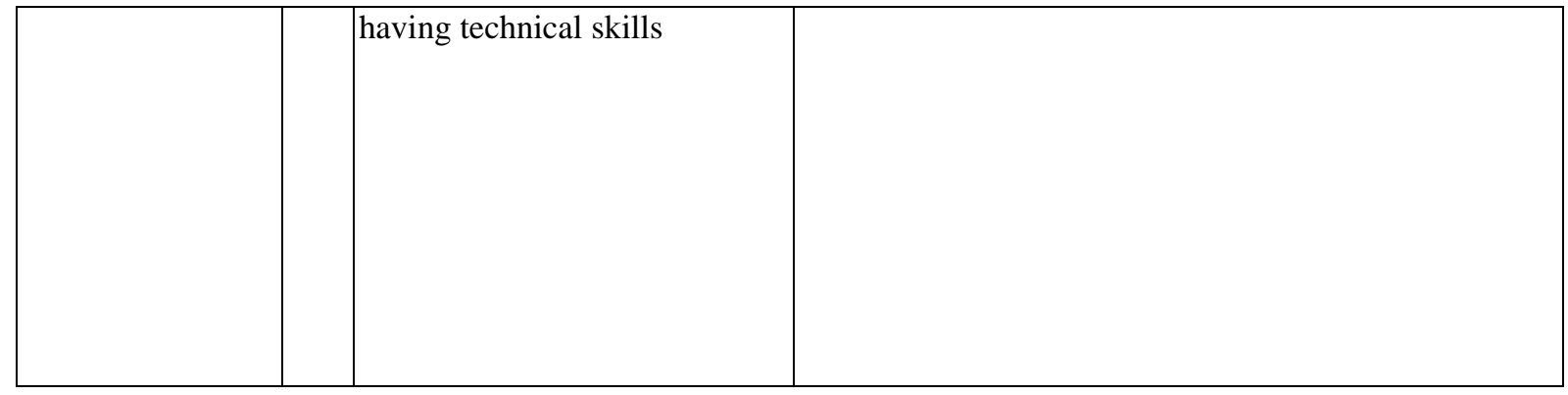

\subsection{Theoretical and Methodological Details}

Table 15 (see Appendix A) presents an overview of the type of system/software/application established, the size and user type forming the sample, and the model tested (in addition to the UTAUT) in each study. It can be seen that TAM (with 29 occurrences) is the most commonly applied model in conjunction with UTAUT, which is followed some way behind by TPB (6 occurrence), TAM2 and the DeLone and McLean IS Success Model (5 occurrences each), IDT and TTF (3 occurrences each), and one occurrence each for TRA, SCT, Trust Model, Andersen's Behavioural Model, and the Theory of Cultural Dimension. It is apparent from Table 15 that the majority of studies used an appropriate sample size, although some employed small samples with fewer than 50 participants (for example, BenMessaoud et al., 2011; Biemans et al., 2005; Lee et al., 2010b, and Samoutis et al., 2008). Some authors (including Chiu and Eysenbach, 2010; Duyck et al., 2008; Hutchison and Bekkering, 2009; Pynoo et al., 2011; van Raaij and Schepers, 2008) acknowledged a small sample size as being one of their limitations, whereas others (including Foon and Fah, 2011; Lee et al., 2010a), in addition to this acknowledgement, also recognized that their limited sample size could hamper generalization of the overall results of their studies. It is worth noting that some studies (Bandyopadhyay and Bandyopadhyay, 2010; Duyck et al., 2010; McLeod et al., 2009a; Pynoo et al., 2008; Pynoo et al., 2011; Yao and Murphy, 2007) utilized more than one sample to test their models, the rationale in some cases (Duyck et al., 2010; Pynoo et al., 2008; Pynoo et al., 2011) being the longitudinal nature of the investigation.

\section{Discussion}

Our intention in this paper was to present the results of a systematic and comprehensive review of the development of UTAUT since its inception in 2003. Based on a review of 174 papers identified from various sources such as Thompson Scientific Web of Science database and Google Scholar, results were presented in terms of six major aspects: demographic characteristics, research topics and types of technology examined, methodological analysis, internal and external variable analysis, analysis of major limitations, and theoretical and methodological details.

Our analysis of the most prolific authors illustrates that the 11 most productive individuals (e.g., Brown et al., 2010; Liu et al., 2008; Weerakkody et al., 2009) in terms of UTAUTbased publications contributed to $13 \%$ of the total number of articles, which is around a quarter of the volume produced by the same number of the most productive authors (see Lee 
et al., 2003; Legris et al., 2003) publishing TAM related research. This indicates that the field currently remains diversified in terms of the number of authors contributing to the UTAUT related articles, with no prominent group of individuals dominating. A similar picture emerges from our analysis of outlets publishing UTAUT research, the field currently being highly diversified with no "obvious" journal or set of journals being the natural home for UTAUT work. This contrasts with the situation pertaining to TAM research, where a number of key journals (including MIS Quarterly and Information \& Management) have attracted a substantial amount of content. The natural and obvious reason for this would be the greater level of maturity of TAM compared to UTAUT, however, some nine years after the appearance of the original UTAUT article by Venkatesh et al. (2003), and despite a fast growing and substantial number of citations, the number of studies published in comparable journals actually making use of UTAUT remains relatively low.

Our country analysis indicates that research in the 174 publications considered was conducted in 41 countries via the activities of researchers affiliated to universities in 36 different countries. The USA was the leading country both in terms of location for research and number of research affiliations. It was noticeable that in a number of cases, numerous authors were affiliated with universities in a particular country, but little primary data has actually been collected in that country - for instance, 38 researchers were affiliated with universities in the UK, yet only two studies were based on primary data collected in the UK. Given the current dominance of the USA as the principal location for collecting primary data (and the limited work conducted elsewhere), there is clearly ample opportunity for researchers to conduct original work by collecting data in additional countries.

Given our analysis examined 174 articles, it was noticeable that there was no leading institution or group of institutions in terms of the number of articles published, with Renmin University in China being the leading institution in terms of number of papers produced (\#5). Despite the USA being by far the preferred location for collecting primary data, and USAbased researchers dominating in terms of the number of articles actually produced, only four USA-based institutions appear in the list of the top 18 universities in terms of number of articles produced, the universities of Nevada and North Texas being placed equal 12th. This indicates that the large amount of UTAUT-related research effort in the USA is spread throughout a large number of contributing institutions, rather than any small number being seen to specialize in such work.

A similar line of enquiry for the most prolific authors also suggests that there is no monopoly of any group of authors in publishing research on UTAUT. Six authors, including Venkatesh, jointly hold the leading position with four articles each, followed by five authors with three articles each. A further 39 authors contributed two articles each, and by far the largest group of 377 authors contributed to just one article each, indicating again that research using UTAUT has been diversified over the years, and no researchers appear to have yet made it their primary area of focus.

Analysis of the most productive departments reveals that most UTAUT work has been carried out by researchers based in departments related to the business, management, information systems and technology fields. This is as might be expected, and we suggest that extended use of UTAUT in additional and diverse fields of study (we have currently seen some limited use in journalism, psychology, education and medicine) is likely to augment the 
level of understanding of the value of the theory, along with contributing to the identification of further strengths and weaknesses.

Our analysis in terms of publication statistics demonstrates that the number of UTAUTrelated publications appearing has generally increased year upon year since the appearance of the original article, with a significant increase in numbers since 2008. Our results show fewer articles appearing in 2011 due to the timing of our data collection activities, but we anticipate that the upward trend will continue, albeit in relatively modest terms.

Results of our keyword analysis suggest that the model has been primarily used for technology adoption and acceptance research in the areas of e-government, e-banking, elearning and e-commerce. In terms of the eight contributing theories and models, TAM has been most often discussed alongside UTAUT. Our findings reveal that office systems have attracted little investigative attention from researchers making use of UTAUT, which is in direct contrast to the findings of Lee et al. (2003) in their study of TAM, in which the study of office systems accounted for $27 \%$ of cases. This situation is essentially a comment on the evolution of systems that are of interest to researchers - clearly in the period covered by Lee et al. (2003), office systems were deemed worthy of investigation, whereas they are now commonplace, and not viewed as being a particularly new technology in the organisational environment. The relatively recent widespread introduction and use of customer-facing technology in domains such as government, retailing, and education has seen a range of new opportunities for original research emerge and continue to materialize, and there is still ample opportunity for researchers to conduct innovative work.

In terms of the methodological aspects of UTAUT research, our investigation revealed a very similar set of results to those of Lee et al. (2003) in that despite the acknowledged value of longitudinal studies in investigating users' changing attitudes toward technology over time as they become familiarized (Doll and Ahmed, 1983), only a minority of studies have been longitudinal in nature, with by far the majority of studies making use of a cross-sectional approach. This may be a result of the relatively recent emergence of UTAUT, but when combined with the dominance of the survey approach, it can be seen that there remains ample scope for original research beyond the current cross-sectional/survey dominance by making use of alternative methodological contexts, tools and techniques. According to Lee et al. (2003) in their study of TAM research, field study and lab experiment were the most common approaches, whereas in our examination of UTAUT research, they appear to have been little used thus far.

PLS and regression analysis have been commonly used in both TAM and UTAUT-based studies, and while other techniques such as SEM, CFA, and FA have been frequently employed to date in UTAUT research, they have been used to a far lesser extent in the TAM context. This may be a reflection on the gradual evolution of methodological preferences, or may be accounted for by other reasons - hence this aspect and other methodological issues would appear to be worthy of further investigation. In terms of software tools used to support analysis during UTAUT investigations, SPSS currently appears to be most favoured, while AMOS, LISREL, and PLS Graph have also been used on a number of occasions. This overall situation is again in contrast to the findings of Lee et al. (2003) in which analysis in TAM studies was generally carried out using LISREL. 
A reasonably large $(22.5 \%)$ contribution of data collected during the UTAUT research considered in our study came from students, and while it is acknowledged that such data samples may not always be representative of the situation in the "real world" (Dwivedi et al., 2008), it does reflect that the approach remains a relatively convenient way for academic researchers to capture data.

Of the 102 quantitative studies using theories and theoretical constructs, 32 made use of more than one model in the same study to differentiate between aspects of research in terms of models, user types, and sector types. This use of multiple models within a single study expands the number of results we are able to consider in our investigation from 102 to 159, and hence increased the amount of input into our analysis of the overall performance of the theory and its constituent relationships. Results from our corresponding weight analysis between sets of relationships indicated that only PE and BI met the requirements of Jeyaraj et al. (2006) to be classed as best predictors of BI and Use Behavior respectively. Hence, there is still a need for further work in this respect in order to examine the role played by other variables, and their potential to qualify for the best predictor category.

From our diagrammatic representation of the diverse range of external variables examined by various studies (see Figure 3), it can be seen that the largest group of variables was examined in terms of their influence on the behavioral intention construct. This is entirely as would be expected given the intention of UTAUT to assist with the measurement of the intention to adopt a new technology. Our analysis of the most frequently external constructs indicated that aspects viewed by Venkatesh et al. (2003) as being accounted for and measured as part of the original UTAUT model often also appear as external variables in published UTAUT-related research - in essence, such cases see the variables concerned as being measured to a greater extent than anticipated. Our results in this respect mirror to an extent those of Lee et al. (2003) in their study of TAM research that also identified certain external variables being accounted for to a greater or lesser extent by TAM itself.

Limitations acknowledged by the studies included in our investigation appear to center on data collection issues - such as focusing on single subject or single task, conducting investigations that are cross sectional in nature and those which are limited in sample size. Self-reporting of actual use was also an issue, but not to the same extent as reported by Lee $e t$ al. (2003) in their study of TAM research, which identified self-reporting of system use as the main weakness. There would therefore appear to be much scope for researchers to conduct original work that addresses these reported limitations.

\section{Conclusion, Limitations, and Future Work}

Our intention in this paper is to present an overview of the current state of UTAUT-related research by presenting the results of a systematic and comprehensive review of 174 articles appearing since 2004. Results were presented in terms of six major aspects: demographic characteristics, research topics and types of technology examined, methodological analysis, internal and external variable analysis, analysis of major limitations, and theoretical and methodological details. Our intent in conducting the investigation was to provide a useful and usable resource for future researchers by providing information on the key areas previously addressed in UTAUT research, how UTAUT research tends to be carried out, and what is usually studied during the course of UTAUT research. 
In keeping with previous 'state of play' studies of this nature, we posit that our findings highlight promising lines of inquiry as well as those that are neglected and those that have already received much attention. All three aspects of analysis in our study imply that UTAUT research is still in its relatively early stages of development, with no clear areas of maturity, but appears to be developing quickly. UTAUT has evolved and been tested and augmented by researchers making use of existing models in conjunction with UTAUT, and by introducing variables and exploring alternative relationships between its constituent components in various contexts and environments, but there are still ample and clear opportunities for researchers to engage with and further shape and develop the field.

Our results reveal that there are many journals and conferences publishing UTAUT research, with contributors from many regions although the majority is unsurprisingly from the USA. There are therefore many opportunities for researchers from other regions to embark on original studies of culture and context-related UTAUT research. The acknowledged limitations of published work provide an initial point from which to identify areas suitable for further research - overly focused subjects and tasks, limited sample sizes in some studies, and a lack of longitudinal work all provide indicators to further opportunities for researchers. Self-reported usage, use of student samples, and a lack of consideration of moderating variables also suggest areas where additional work can be viewed as being necessary. Finally, the results of our weight analysis suggest that the cumulative predictive power of each individual independent variable was not consistent or at the level expected, with only two variables (performance expectancy and behavioural intention) meeting the benchmark of Jeyaraj et al. (2006) and qualifying for best predictor category. Further investigation into the performance of the relationships within the model would therefore appear to be appropriate.

We anticipate this paper will prove to be a useful source of information for those readers who wish to learn more about the various facets pertaining to published UTAUT research, and suggest that the findings of this study may help in directing limited and valuable research resources to potentially fruitful lines of inquiry as well as strengthening the overall field of UTAUT research by facilitating consideration of useful alternative theoretical and methodological perspectives, and by highlighting aspects requiring further scrutiny. However, we acknowledge that our study has a number of limitations and readers should interpret the material presented in this paper within the context of these limitations.

Perhaps the most obvious limitation is that of literature forming our sample - as with all articles of this type, our results reflect the material actually examined, and clearly there may be significant and influential work that we have not included. For instance, our search activities were centered on occurrences of keywords in order to avoid locating large numbers of publications where these keywords might have been used as casual words in the main text. We fully acknowledge that there may be numerous studies, which lack keywords in the title, but still focus upon UTAUT in some form. We admit this aspect and encourage further research to extend the amount of material considered. However, we posit that our sampling approach was sufficient to provide a representative reflection of the current state of UTAUT research.

\section{References}


AbuShanab, E. and Pearson, J.M. (2007), "Internet banking in Jordan: The unified theory of acceptance and use of technology (UTAUT) perspective", Journal of Systems and Information Technology, Vol. 9, No. 1, pp. 78-97.

Abu-Shanab, E. and Pearson, J.M. (2009), "Internet Banking in Jordan: An Arabic Instrument Validation Process", International Arab Journal of Information Technology, Vol. 6, No. 3, pp. 235244.

Abu-Shanab, E., Pearson, J.M. and Setterstrom, A.J. (2010), "Internet Banking and Customers' Acceptance in Jordan: The Unified Model's Perspective", Communications of the Association for Information Systems, Vol. 26, pp. 493-524.

Agarwal, R. and Karahanna, E. (2000), "Time files when you are having fun: Cognitive absorption and beliefs about information technology usage", MIS Quarterly, Vol. 24, No. 4, pp. 665-694.

Aggelidis, V.P. and Chatzoglou, P.D. (2009), "Using a modified technology acceptance model in hospitals", International Journal of Medical Informatics, Vol. 78, No. 2, pp. 115-126.

Alapetite, A., Andersen, H.B. and Hertzum, M. (2009), "Acceptance of speech recognition by physicians: A survey of expectations, experiences, and social influence", International Journal of Human-Computer Studies, Vol. 67, No. 1, pp. 36-49.

Al Awadhi, S. and Morris, A. (2011), "The use of the UTAUT model in the adoption of e-government services in Kuwait", Proceedings of the 41 st Hawaii International Conference on System Sciences.

Al-Gahtani, S.S., Hubona, G.S. and Wang, J. (2007), "Information technology (IT) in Saudi Arabia: Culture and the acceptance and use of IT", Information \& Management, Vol. 44, No. 8, pp. 681-691.

Al-Harby, F., Qahwaji, R. and Kamala, M. (2010), "Users' Acceptance of Secure Biometrics Authentication System: Reliability and Validate of an Extended UTAUT Model", Communications in Computer and Information Science, Vol. 87, No. 2, pp. 254-258.

Al-Rajhi, M., Liu, K. and Nakata, K.A. (2010), "Conceptual Model for Acceptance of Information Systems: An Organizational Semiotic Perspective”, AMCIS 2010 Proceedings.

Al-Shafi, S. and Weerakkody, V. (2009), "Understanding Citizens' Behavioural Intention in the Adoption of E-Government Services in the State of Qatar", European Conference on Information System.

Al-Shafi, S. and Weerakkody, V. (2010), "Factors affecting e-government adoption in the State of Qatar", European and Mediterranean Conference on Information Systems.

Al-Sobhi, F., Weerakkody, V. and El-Haddadeh, R. (2011), "The roles of intermediaries in egovernment adoption: The case of Saudi-Arabia", tGov Workshop, London.

Al-Somali, S.A., Gholami, R. and Clegg, B. (2009), "An investigation into the acceptance of online banking in Saudi Arabia", Technovation, Vol. 29, No. 2, pp. 130-141.

Ambali, A.R. (2009), "E-Government Policy: Ground issues in e-filling system", European Journal of Social Sciences, Vol. 11, No. 2, pp. 249-266.

Anderson, J.E., Schwager, P.H. and Kerns, R.L. (2006), "The drivers of acceptance of Tablet PCs by Faculty in a College of Business", Journal of Information Systems Education, Vol. 17, No. 4, pp. 429440.

Aoun, C., Vatanasakdakul, S. and Li, Y. (2010), “AIS in Australia: UTAUT application and cultural implication", ACIS 2010 Proceedings.

Avdic, A. and Eklund, A. (2010), "Searching reference databases: What students experience and what teachers believe that students experience", Journal of Librarianship and Information Science, Vol. 42, No. 4, pp. 224-235.

Bandura, A. (1997), Social Learning Theory. Englewood Cliffs, NJ: Prentice Hall.

Bandyopadhyay, K. and Bandyopadhyay, S. (2010), "User acceptance of information technology across cultures", International Journal of Intercultural Information Management, Vol. 2, No. 3, pp. 218-231. 
Barati, S. and Mohammadi, S. (2009), "An Efficient Model to Improve Customer Acceptance of Mobile Banking”, 2009 World Congress on Engineering and Computer Science.

Barnes, S. and Vidgen, R. (2009), "An evaluation of user acceptance of a corporate intranet", Proceedings of European Conference on Information Systems.

Bauer, R., (1960), Consumer behavior as risk taking, In: D. Cox (Ed.), Risk Taking and Information Handling in Consumer Behavior, Harvard University Press, Cambridge, MA.

BenMessaoud, C., Kharrazi, H. and MacDorman, K.F. (2011), "Facilitators and Barriers to Adopting Robotic-Assisted Surgery: Contextualizing the Unified Theory of Acceptance and Use of Technology", PloS One, Vol. 6, No. 1.

Benslimane, Y., Plaisent, M. and Bernard, P. (2004), "Using Web Systems for eProcurement: An Extension of the Unified Theory of Acceptance and Use of Technology", Proceedings of European Conference on Information Systems.

Biemans, M., Swaak, J., Hettinga, M. and Schuurman, J.G. (2005), "Involvement Matters: The Proper Involvement of Users and Behavioural Theories in the Design of a Medical Teleconferencing Application", Proceedings of the International ACM SIGGROUP Conference on Supporting Group Work, pp. 304-312.

Brown, S.A., Dennis, A.R. and Venkatesh, V. (2010), "Predicting Collaboration Technology Use: Integrating Technology Adoption and Collaboration Research", Journal of Management Information Systems, Vol. 27, No. 2, pp. 9-53.

Brown, S.A. and Venkatesh, V. (2005), "Model of Adoption of Technology in Households: A Baseline Model Test and Extension Incorporating Household Life Cycle", MIS Quarterly, Vol. 29, No. 3, pp. 399-426.

Butler, J. and Richardson, K. (2005), "The perceived ease of use of iBrainz technology in Property Law and Taxation Law", Studies in Learning, Evaluation, Innovation and Development: Women in Research, Contemporary Issues in Research (A), Vol. 81.

Cabral, C., Lucas, P. and Gordon, D. (2009), "Technology adoption in developing country contexts: testing water for microbial contamination", Aquatest Working Paper.

Carlsson, C., Carlsson, J., Hyvonen, K., Puhakainen, J. and Walden, P. (2006), “Adoption of Mobile Devices/Services - Searching for Answers with the UTAUT", Proceedings of the 39th Hawaii International Conference on System Sciences, pp. 1-10.

Carter, L. and Schaupp, L.C. (2009), "Relating Acceptance and Optimism to E-File Adoption", International Journal of Electronic Government Research, Vol. 5, No. 3, pp. 62-74.

Chan, F.K.Y., Thong, J.Y.L., Venkatesh, V., Brown, S.A., Hu, P.J-H. and Tam, K.Y. (2010), "Modeling Citizen Satisfaction with Mandatory Adoption of an E-Government Technology", Journal of the Association for Information Systems, Vol. 11, No. 10, pp. 519-549.

Chang, I-C., Hwang, H-G., Hung, W-F. and Li, Y-C. (2007), "Physicians' acceptance of pharmacokinetics-based clinical decision support systems", Expert Systems with Applications, Vol. 33, No. 2, pp. 296-303.

Chen, C., Wu, J. and Yang, S. (2008), "Accelerating the Use of Weblogs as an Alternative Method to Deliver Case-Based Learning”, International Journal on E-Learning, Vol. 7, No. 2, pp. 331-349.

Cheng, D., Liu, G. and Qian, C. (2008a), "On Determinants of User Acceptance of Internet Banking: A Theoretical Framework and Empirical Study", IEEE Symposium on Advanced Management of Information for Globalized Enterprises.

Cheng, D., Liu, G., Qian, C. and Song, Y.F. (2008b), "Customer Acceptance of Internet Banking: Integrating Trust and Quality with UTAUT Model", IEEE International Conference on Service Operations and Logistics, and Informatics.

Cheng, D., Liu, G., Song, Y.F. and Qian, C. (2008c), “Adoption of Internet Banking: An Integrated Model. $4^{\text {th }}$ International Conference on Wireless Communications", Networking and Mobile 
Computing, Vol. 1, No. 31, pp. 65-68.

Chisolm, D.J., Purnell, T.S., Cohen, D.M. and McAlearney, A.S. (2010), "Clinician Perceptions of an Electronic Medical Record During the First Year of Implementation in Emergency Services", Pediatric Emergency Care, Vol. 26, No. 2, pp. 107-110.

Chiu, C.M. and Wang, E.T.G. (2008), "Understanding Web-based learning continuance intention: The role of subjective task value", Information \& Management, Vol. 45, No. 3, pp. 194-201.

Chiu, C.M., Huang, H.Y. and Yen, C.H. (2010), "Antecedents of trust in online auctions", Electronic Commerce Research and Applications, Vol. 9, No. 2, pp. 148-159.

Chiu, T.M.L, \& Eysenbach, G. (2010), "Stages of use: consideration, initiation, utilization, and outcomes of an internet-mediated intervention", BMC Medical Informatics and Decision Making, Vol. 10, pp. 73, pp. 1-11.

Cody-Allen, E. and Kishore, R. (2006), "An extension of the UTAUT model with e-quality, trust, and satisfaction constructs", Proceedings of the ACM SIGMIS CPR Conference on Computer Personnel Research.

Compeau, D.R. and Higgins, C.A., (1995), "Computer self-efficacy: Development of a measure and initial test”, MIS Quarterly, Vol. 19, No. 2, pp. 189-211.

Cornacchia, M., Baroncini, V. and Livi, S. (2008), "Predicting the influence of emerging information and communication technologies on home life", Lecture Notes in Business Information Processing, Vol. 8, pp. 184-200.

Coss, D.L. (2009), "Individual's intention to use self-diagnostic medical support system", Southern Association for Information Systems Conference, pp. 1-6.

Curtis, L., Edwards, C., Fraser, K.L., Gudelsky, S., Holmquist, J., Thornton, K. and Sweetser, K.D. (2010), "Adoption of social media for public relations by non-profit organizations", Public Relations Review, Vol. 36, No. 1, pp. 90-92.

Curtis, M.B. and Payne, E.A. (2008), "An examination of contextual factors and individual characteristics affecting technology implementation decisions in auditing", International Journal of Accounting Information Systems, Vol. 9, No. 1, pp. 104-121.

Dada, D. (2006), "E-readiness for developing countries: Moving the focus from the environment to the users", The Electronic Journal on Information Systems in Developing Countries, Vol. 27, No. 6, pp. 1-14.

Dadayan, L. and Ferro, E. (2005), "When technology meets the mind: A comparative study of the technology acceptance model", In M.A. Wimmer, R. Traunmuller, A. Gronlund and K.V. Andersen (Eds.), Electronic Government Proceedings, Vol. 3591, pp. 137-144.

Dasgupta, S. and Gupta, B. (2010), "Organizational Culture and Technology Use in a Developing Country: An Empirical Study", SIG GlobDev Third Annual Workshop.

Davis, F.D. (1989), "Perceived usefulness, perceived ease of use, and user acceptance of information technology", MIS Quarterly, Vol. 13, No. 3, pp. 319-340.

Davis, F.D., Bagozzi, R.P. and Warshaw, P.R. (1992), "Extrinsic and intrinsic motivation to use computers in the workplace", Journal of Applied Social Psychology, Vol. 22, No. 14, pp. 1111-1132. de Silva, H. and Ratnadiwakara, D. (2009), "Social Influence in Mobile Phone Adoption: Evidence from bottom of Pyramid in Emerging Asia", Paper presented at Mobile 2.0: Beyond Voice Preconference workshop at the International Communication Association (ICA) Conference, Chicago, Illinois.

Debuse, J.C.W., Lawley, M. and Shibl, R. (2008), "Educators' perceptions of automated feedback systems", Australasian Journal of Educational Technology, Vol. 24, No. 4, pp. 374-386.

DeLone, W.H. and McLean, E.R. (1992), "Information systems success: The quest for the dependent variable", Information Systems Research, Vol. 3, No. 1, pp. 60-95. 
Diaz, M.C. and Loraas, T. (2010), "Learning new uses of technology while on an audit engagement: Contextualizing general models to advance pragmatic understanding", International Journal of Accounting Information Systems, Vol. 11, pp. 61-77.

Doll, W.J. and Ahmed, M.U. (1983), "Managing User Expectations", Journal of Systems Management, Vol. 34, No. 6, pp. 6-11.

Dulle, F.W. and Minishi-Majanja, M.K. (2011), "The suitability of the Unified Theory of Acceptance and Use of Technology (UTAUT) model in open access adoption studies", Information Development Vol. 27, No. 1, pp. 32-45.

Duyck, P., Pynoo, B., Devolder, P., Voet, T., Adang, L. and Ovaere, D. (2010), "Monitoring the PACS Implementation Process in Large University Hospital-Discrepancies between Radiologists and Physicians", Journal of Digital Imaging, Vol. 23, No. 1, pp. 73-80.

Duyck, P., Pynoo, B., Devolder, P., Voet, T., Adang, L. and Vercruysse, J. (2008), "User acceptance of a Picture Archiving and Communication System - Applying the unified theory of acceptance and use of technology in a radiological setting", Methods of Information in Medicine, Vol. 47, No. 2, pp. 149-156.

Dwivedi, Y.K., Kiang, M.Y., Williams, M.D. and Lal, B. (2008), "Profiling research published in the Journal of Electronic Commerce Research", Journal of Electronic Commerce Research, Vol. 9, No. 2, pp. 77-91.

Fishbein, M. and Ajzen, I. (1975), Belief, attitude, intention, and behavior: An introduction to theory and research. Reading Mass: Addison-Wesley.

Fitterer, R., Rohner, P., Mettler, T. and Winter, R. (2010), “A taxonomy for multi-perspective ex-ante evaluation of the value of complementary health information systems - applying the unified theory of acceptance and use of technology", Proceedings of the $43^{\text {rd }}$ Hawaii International Conference on System Sciences, pp. 1-10.

Foon, Y.S. and Fah, B.C.Y. (2011), "Internet Banking Adoption in Kuala Lumpur: An Application of UTAUT Model”, International Journal of Business and Management, Vol. 6, No. 4, pp. 161-167.

Gunther, O., Krasnova, H., Riehle, D. and Schonberg, V. (2009), "Modeling Micro-Blogging Adoption in the Enterprise", In Proceedings of the Fifteenth Americas Conference on Information Systems, San Francisco.

Gupta, B., Dasgupta, S. and Gupta, A. (2008), "Adoption of ICT in a government organization in a developing country: An empirical study”, Journal of Strategic Information Systems, Vol. 17, No. 2, pp. 140-154.

Hailemariam, G., Negash, S. and Musa, P.F. (2010), "In search of Insights from Community of Practice and Use of Telemedicine in Low income Countries: The case of Ethiopia", AMCIS Proceedings, pp. 1-11.

He, D.H. and Lu, Y.B. (2007a), "Consumers Perceptions and Acceptances towards Mobile Advertising: An Empirical Study in China", International Conference on Wireless Communications, Networking and Mobile Computing.

He, D.H. and Lu, Y.B. (2007b), "An integrated framework for mobile business acceptance", The Sixth Wuhan International Conference on E-Business, pp. 305-313.

He, W. and Wei, K-K. (2009), "What drives continued knowledge sharing? An investigation of knowledge-contribution and -seeking beliefs" Decision Support Systems, Vol. 46, pp. 826-838.

Heerink, M., Krose, B., Wielinga, B. and Evers, V. (2009), "Measuring the influence of social abilities on acceptance of an interface robot and a screen agent by elderly users", In Proceedings of British Computer Society Conference on HCI, pp. 430-439.

Heikkila, J-P. and Smale, A. (2010), "The effects of 'language standardization' on the acceptance and use of e-HRM systems in foreign subsidiaries", Journal of World Business, pp. 1-9. 
Hennington, A., Janz, B., Amis, J. and Nichols, E. (2009), "Information Systems and Healthcare XXXII: Understanding the Multidimensionality of Information Systems Use: A Study of Nurses' Use of a Mandated Electronic Medical Record System", Communications of the Association for Information Systems, Vol. 25, No. 1, pp. 243-262.

Ho, C-T.B. and Chou, U-T.T. (2009), "The Critical Factors for Applying Podcast in Mobile Language Learning", International Conference on Information Management and Engineering, pp. 411-415.

Huang, K-Y., Choi, N. and Chengalur-Smith, I. (2010), "Cultural Dimensions as Moderators of the UTAUT Model: A Research Proposal in a Healthcare Context", Americas Conference on Information Systems, pp. 1-9.

Huang, S. and Wang, X. (2009), "Influence of Organizational System to End-Users' Acceptance of ERP System in Chinese Enterprises", Ninth International Conference on Hybrid Intelligent Systems, pp. 160-164.

Hung, Y.H., Wang, Y.S. and Chou, S.C.T. (2007), "User Acceptance of E-Government Services", Pacific Asia Conference on Information Systems, Kaohsiung: Natl Sun Yat-Sen University.

Huser, V., Narus, S.P. and Rocha, R.A. (2010), "Evaluation of a flowchart-based EHR query system: A case study of Retro Guide", Journal of Biomedical Informatics, Vol. 43, No. 1, pp. 41-50.

Hutchison, D. and Bekkering, E. (2009), "Using Remote Desktop Applications in Education, Information", Systems Education Journal, Vol. 7, No. 13, pp. 1-13.

Im, I., Kim, Y. and Han, H-J. (2008), "The effects of perceived risk and technology type on users' acceptance of technologies", Information \& Management, Vol. 45, No. 1, pp. 1-9.

Jalaldeen, R., Karim, N. and Mohamed, N. (2009), "Organizational Readiness and its Contributing Factors to Adopt KM Processes: A Conceptual Model”, Communications of the IBIMA, Vol. 8, No. 17, pp. 128-136.

Jayasingh, S. and Eze, U.C. (2009), "An Empirical Analysis of Consumer Behavioral Intention toward Mobile Coupons in Malaysia", International Journal of Business and Information, Vol. 4, No. 2, pp. 221-242.

Jeyaraj, A., Rottman, J.W. and Lacity, M.C. (2006), "A review of the predictors, linkages, and biases in IT innovation adoption research", Journal of Information Technology, Vol. 21, No. 1, pp. 1-23.

Johari, M.H.Y., Roni, M.S.M.M., Ahmad, A. and Hasim, A.K.M. (2010), "Visitors' use of information kiosk at Melaka town heritage sites", Business Management Quarterly Review, Vol. 1, No. 2, pp. 54-67.

Johnston, A.C. and Warkentin, M. (2010), "Fear appeals and information security behaviors: An empirical study", MIS Quarterly, Vol. 34, No. 3, pp. 549-566.

Jong, D. and Wang, T.S. (2009), Student Acceptance of Web-based Learning System. Oulu: Academic Publication.

Keller, C., Hrastinski, S. and Carlsson, S.A. (2007), "Students' Acceptance of E-learning Environments: A Comparative Study in Sweden and Lithuania", Proceedings of the $15^{\text {th }}$ European Conference on Information Systems, pp. 395-406.

Kijsanayotin, B., Pannarunothai, S. and Speedie, S.M. (2009), "Factors influencing health information technology adoption in Thailand's community health centres: Applying the UTAUT model", International Journal of Medical Informatics, Vol. 78, No. 6, pp. 404-416.

Koh, C.E., Prybutok, V.R., Ryan, S.D. and Wu, Y.A. (2010), "A Model for Mandatory Use of Software Technologies: An Integrative Approach by Applying Multiple Levels of Abstraction of Informing Science", The International Journal of an Emerging Transdiscipline, Vol. 13, pp. 177-203. Koivumaki, T., Ristola, A. and Kesti, M. (2008), "The perceptions towards mobile services: An empirical analysis of the role of use facilitators", Personal and Ubiquitous Computing, Vol. 12, No. 1, pp. 67-75. 
Kourouthanassis, P.E., Georgiadis, C., Zamani, E. and Giaglis, G.M. (2010), "Explaining the Adoption of Mobile Internet Applications", $9^{\text {th }}$ International Conference on Mobile Business, pp. 148153.

Laumer, S., Eckhardt, A. and Trunk, N. (2010), "Do as your parents say?-Analyzing IT adoption influencing factors for full and under age applicants", Information Systems Frontiers, Vol. 12, No. 2, pp. 169-183.

Lee, C-L., Yen, D.C., Peng, K-C. and Wu, H-C. (2010a), "The influence of change agents' behavioral intention on the usage of the activity based costing/management system and firm performance: The perspective of unified theory of acceptance and use of technology", Advances in Accounting, incorporating Advances in International Accounting, Vol. 26, No. 2, pp. 314-324.

Lee, V. and Lin, S-J. (2008), "Podcasting Acceptance on Campus: An extension of the UTAUT Model", DIGIT Proceedings.

Lee, W.Y., Goodwin, P., Fildes, R., Nikolopoulos, K. and Lawrence, M. (2007), "Providing support for the use of analogies in demand forecasting tasks", International Journal of Forecasting, Vol. 23, No. 3, pp. 377-390.

Lee, Y., Kozar, K.A. and Larsen, K.R.T. (2003), "The Technology Acceptance Model: Past, Present, and Future", Communications of the Association for Information System, Vol. 12, No. 1, pp. 752-780. Lee, Y-C., Li, M-L., Yen, T-M. and Huang, T-H. (2010b), "Analysis of adopting an integrated decision making trial and evaluation laboratory on a technology acceptance model", Expert Systems with Applications, Vol. 37, No. 2, pp. 1745-1754.

Legris, P., Ingham, J. and Collerette, P. (2003), "Why do people use information technology? A critical review of the technology acceptance model", Information \& Management, Vol. 40, No. 3, pp. 191-204.

Li, J.P. and Kishore, R. (2006), "How Robust is the UTAUT Instrument? A Multigroup Invariance Analysis in the Context of Acceptance and Use of Online Community Weblog Systems", SIGMISCPR'06, pp. 183-189.

Li, W. (2010), "Virtual knowledge sharing in a cross-cultural context", Journal of Knowledge Management, Vol. 14, No. 1, pp. 38-50.

Lin, J., Chan, H. and Jin, Y. (2004), "Instant Messaging Acceptance and Use among College Students", Pacific Asia Conference on Information Systems, pp. 181-194.

Liu, G., Huang, S-P. and Zhu, X-K. (2008), "User acceptance of Internet banking in an uncertain and risky environment", The 2008 International Conference on Risk Management \& Engineering Management.

Loo, W.H., Yeow, P.H.P. and Chong, S.C. (2009), "User acceptance of Malaysian government multipurpose smartcard applications", Government Information Quarterly, Vol. 26, No. 2, pp. 358367.

Louho, R., Kallioja, M. and Oittinen, P. (2006), "Factors affecting the Use of Hybrid Media Applications", Graphic Arts in Finland, Vol. 35, No. 3, pp. 11-21.

Lubrin, E., Lawrence, E., Zmijewska, A., Navarro, K.F. and Culjak, G. (2006), "Exploring the Benefits of Using Motes to Monitor Health: An Acceptance Survey", Proceedings of the First International Conference on Mobile Communications and Learning, Mauritius.

Luo, X., Li, H., Zhang, J. and Shim, J.P. (2010), "Examining multi-dimensional trust and multifaceted risk in initial acceptance of emerging technologies: An empirical study of mobile banking services", Decision Support Systems, Vol. 49, No. 2, pp. 222-234.

Mahzan, N. and Lymer, A. (2008), "Adoption of Computer Assisted Audit Tools and Techniques (CAATTs) by Internal Auditors: Current issues in the UK", BAA Annual Conference.

Maldonado, U.P.T., Khan, G.F., Moon, J. and Rho, J.J. (2009), "E-learning motivation, Students' Acceptance/Use of Educational Portal in Developing Countries", Fourth International Conference on 
Computer Sciences and Convergence Information Technology.

Maldonado, U.P.T., Khan, G.F., Moon, J. and Rho, J.J. (2011), "E-learning motivation and educational portal acceptance in developing countries", Online Information Review, Vol. 35, No. 1, pp. 66-85.

Marchewka, J., Liu, C. and Kostiwa, K. (2007), "An Application of the UTAUT Model for Understanding Student Perceptions Using Course Management Software", Communications of the IIMA, Vol. 7, No. 2, pp. 93-104.

Mayer, P., Volland, D., Thiesse, F. and Fleisch, E. (2011), "User Acceptance of 'Smart Products': An Empirical Investigation", Wirtschaftinformatik Proceedings, pp. 1063-1072.

Mayers, R.C., Davis, J.H. and Schoorman, F.D. (1995), "An integrative model of organizational trust", Academy of Management Review, Vol. 20, No. 3, pp. 709-734.

McKnight, D.H. and Chervany, N.L. (2002), "What trust means in e-commerce customer relationships: An interdisciplinary conceptual typology", International Journal of Electronic Commerce, Vol. 6, No. 2, pp. 296-315.

McLeod, A., Pippin, S. and Catania, V. (2009a), "Using Technology Acceptance Theory to Model Individual Differences in Tax Software Use", Americas Conference on Information Systems, pp. 1-11. McLoad, A., Pippin, S. and Mason, R. (2009b), "Individual Taxpayer Intention to Use Tax Preparation Software: Examining Experience, Trust, and Perceived Risk", Journal of Information Science and Technology, Vol. 6, No. 1, pp. 25-44.

Moore, G.C. and Benbasat, I., (1991), "Development of an instrument to measure the perceptions of adopting an information technology innovation", Information Systems Research, Vol. 2, No. 3, pp. 192-222.

Neufeld, D.J., Dong, L.Y. and Higgins, C. (2007), "Charismatic leadership and user acceptance of information technology", European Journal of Information Systems, Vol. 16, No. 4, pp. 494-510.

Niehaves, B. and Plattfaut, R. (2010), "What Is the Issue with Internet Acceptance among Elderly Citizens? Theory Development and Policy Recommendations for Inclusive E-Government", In M.A. Wimmer, J.L. Chappelet, M. Janssen and H. J. Scholl (Eds.), Electronic Government, Vol. 6228, pp. 275-288.

Nistor, N., Wagner, M., Istvanffy, E. and Dragota, M. (2010), “The Unified Theory of Acceptance and Use of Technology: Verifying the model from a European perspective", International Journal of Knowledge and Learning, Vol. 6, No. 2-3, pp. 185-199.

Nov, O. and Ye, C. (2009), "Resistance to Change and the Adoption of Digital Libraries: An Integrative Model", Journal of the American Society for Information Science and Technology, Vol. 60, No. 8, pp. 1702-1708.

Or, C.K.L., Karsh, B-T., Severtson, D.J., Burke, L.J., Brown, R.L. and Brennan, P.F. (2011), "Factors affecting home care patients' acceptance of a web-based interactive self-management technology", Journal of American Medical Informatics Association, Vol. 18, No. 1, pp. 51-59.

Oshlyansky, L., Cairns, P. and Thimbleby, H. (2007), "Validating the Unified Theory of Acceptance and Use of Technology (UTAUT) tool cross-culturally", The $21^{\text {st }}$ British HCI Group Annual Conference.

Pahlke, I. and Beck, R. (2009), "Assimilation of Enterprise Mashup Systems - The Impact on Changes in Work Processes", DIGIT Proceedings.

S. Pahnila, M. Siponen, Myyry, L. and Zheng, X. (2011), "The influence of individualistic and collectivistic values to UTAUT: The case of the Chinese eBay", European Conference on Information Systems.

Pai, J.C. and Tu, F.M. (2011), "The acceptance and use of customer relationship management (CRM) systems: An empirical study of distribution service industry in Taiwan", Expert Systems with Applications, Vol. 38, No. 1, pp. 579-584. 
Pappas, F.C. and Volk, F. (2007), "Audience counts and reporting system: Establishing a cyberinfrastructure for museum educators", Journal of Computer-Mediated Communication, Vol. 12, No. 2, pp. 752-768.

Park, J., Yang, S. and Lehto, X. (2007), "Adoption of mobile technologies for Chinese consumers", Journal of Electronic Commerce Research, Vol. 8, No. 3, pp. 196-206.

Pavon, F. and Brown, I. (2010), "Factors influencing the adoption of the World Wide Web for jobseeking in South Africa", South African Journal of Information Management, Vol. 12, No. 1, pp. 1-9. Payne, K. (2008), "Much Ado about Something: Web 2.0 Acceptance and Use by Public Relations Practitioners", Public Relations Society of America, Detroit, Michigan, USA, pp. 76-92.

Pynoo, B., Devolder, P., Voet, T., Vercruysse, J., Adang, L., Ovaere, D. and Duyck, P. (2008), "How about the end-users? Critical factors during the implementation of PACS", International Journal of CARS, Vol. 3, No. 1, pp. 169-173.

Pynoo, B., Devolder, P., Tondeur, J., van Braak, J., Duyck, W. and Duyck, P. (2011), "Predicting secondary school teachers' acceptance and use of a digital learning environment: A cross-sectional study", Computers in Human Behavior, Vol. 27, No. 1, pp. 568-575.

Qingfei, M., Shaobo, J. and Gang, Q. (2008), "Mobile Commerce User Acceptance Study in China: A Revised UTAUT Model”, Tsinghua Science and Technology, Vol. 13, No. 3, pp. 257-264.

Randeree, E. (2009), "Investigating literacy as a predictor of adoption and usage", Proceedings of the Southern Association for Information Systems Conference, Charleston, SC, pp. 152-157.

Reunis, M. and Santema, S. (2005), "Achieving Individual Adoption of E-Ordering Applications with a 'Benefit Imbalance'”, Proceedings of RSEEM, pp. 203-219.

Richardson, K., Butler, J. and Holm, E. (2009), "Teaching law to non-law students: The use of problem solving models in legal teaching", Studies in Learning, Evaluation Innovation and Development, Vol. 6, No. 2, pp. 29-41.

Rogers, E.M. (1983), Diffusion of Innovations. New York, NY: The Free Press.

Sahu, G.P. \& Gupta, M.P. (2007), "Users' Acceptance of E-Government: A study of Indian central Excise", International Journal of Electronic Government, Vol. 3, No. 3, pp. 1-21.

Sambasivan, M., Wemyss, G.P. and Rose, R.C. (2010), "User acceptance of a G2B system: A case of electronic procurement system in Malaysia”, Internet Research, Vol. 20, No. 2, pp. 169-187.

Samoutis, G.A., Soteriades, E.S., Stoffers, H.E., Zachariadou, T., Philalithis, A. and Lionis, C. (2008), "Designing a multifaceted quality improvement intervention in primary care in a country where general practice is seeking recognition: The case of Cyprus", BMC Health Services Research, Vol. 8, No. 1, pp. 1-9.

Sapio, B., Turk, T., Cornacchia, M., Papa, F., Nicolo, E. and Livi, S. (2010), "Building scenarios of digital television adoption: A pilot study", Technology Analysis \& Strategic Management, Vol. 22, No. 1, pp. 43-63.

Schaper, L. \& Pervan, G. (2004), "A Model of Information and Communication Technology Acceptance and Utilisation by Occupational Therapists", The IFIP TC8/WG8.3 International Conference, pp. 734-744.

Schaper, L.K. and Pervan, G.P. (2006), "Developing a model of technology acceptance within the Australian healthcare sector", The $10^{\text {th }}$ Pacific Asia Conference on Information Systems, pp. 835-847. Schaupp, L.C., Carter, L. and McBride, M.E. (2010), 'E-file adoption: A study of US taxpayers' intentions", Computers in Human Behavior, Vol. 26, No. 4, pp. 636-644.

Schwarz, A. and Chin, W. (2007), "Looking Forward: Toward an understanding of the nature and definition of IT acceptance", Journal of the Association for Information Systems, Vol. 8, No. 4, pp. 230-243. 
Seymour, L., Makanya, W. and Berrange, S. (2007), 'End-Users' Acceptance of Enterprise Resource Planning Systems: An Investigation of Antecedents", Proceedings of the 6th Annual ISOnEworld Conference, Las Vegas, NV, 2007.

Shamsuddin, N.B. (2009), "Students Perception towards the Implementation of Computer Graphics Technology in Class via Unified Theory of Acceptance and Use of Technology (UTAUT) Model", In H.B. Zaman, P. Robinson, M. Petrou, P. Olivier, H. Schroder and T.K. Shih (Eds.), Visual Informatics: Bridging Research and Practice, Vol. 5857, pp. 886-893.

Sharma, A. and Citurs, A. (2004), "Incorporating Personality into UTAUT: Individual Differences and User Acceptance of IT", Proceedings of the Americas Conference on Information Systems, New York, New York, August, 3348-3353.

Shi, W.W. (2009), “An Empirical Research on Users' Acceptance of Smart Phone Online Application Software", International Conference on Electronic Commerce and Business Intelligence.

Shin, D.H. (2009), "Towards an understanding of the consumer acceptance of mobile wallet", Computers in Human Behavior, Vol. 25, No. 6, pp. 1343-1354.

Shin, D.H. (2010), "MVNO services: Policy implications for promoting MVNO diffusion", Telecommunications Policy, Vol. 34, No. 10, pp. 616-632.

Simonson, M.R., Maurer, M., Torardi, M. and Whitaker, M., (1987), "Development of a standardized test of computer literacy and a computer anxiety index", Journal of Educational Computing Research, Vol. 3, No. 2, pp. 231-247.

Song, Y. and Han, J. (2009), "Is Enjoyment Important? An empirical research on the impact of perceive enjoyment on adoption of new technology", 2009 International Conference on Information Management, Innovation Management and Industrial Engineering, pp. 511-514.

Spielberger, C.D. (1972), "Conceptual and Methodological Issues in Anxiety Research", In C.D. Spielberger, I.G. Sarason (eds.) Anxiety: Current Trends in Theory and Research. New York: Academic Press, pp. 481-494.

Suh, B. and Han, I. (2002), "Effect of Trust on Customer Acceptance of Internet Banking", Electronic Commerce Research and Applications, Vol. 1, No. 3, pp. 247-263.

Suhendra, E.S., Hermana, B. and Sugiharto, T. (2009), "Behavioral Analysis of Information Technology Acceptance in Indonesia Small Enterprises", Anadolu International Conference in Economics, Turkey.

Suki, N.M. and Ramayah, T. (2010), "User Acceptance of the E-Government Services in Malaysia: Structural Equation Modelling Approach", Interdisciplinary Journal of Information, Knowledge, and Management, Vol. 5, No. 1, pp. 395-413.

Sumak, B., Polancic, G. and Hericko, M. (2010), "An Empirical Study of Virtual Learning Environment Adoption Using UTAUT", Second International Conference on Mobile, Hybrid, and On-line Learning, pp. 17-22.

Taksa, I. and Flomenbaum, J.M. (2009), "An Integrated Framework for Research on Cross-Cultural Information Retrieval. $6^{\text {th }}$ International Conference on Information Technology: New Generations, pp. 1367-1372.

Tan, L. and Wu, L. (2010), “An Empirical Study on Factors Affecting Customer Intention in Mobile Commerce Contexts", $2^{\text {nd }}$ International Conference on Multi Media and Information Technology, pp. 310-313.

Tavares, A. and Amaral, L. (2010), "Adoption of Authenticated Peer-to-Peer Academic Networks - A Case Study of a Failure”, CENTERIS 2010, Part II, CCIS 110, pp. 345-354.

Teo, T. (2011), "Factors influencing teachers' intention to use technology: Model development and test", Computers \& Education, pp. 1-25.

Terzis, V. and Economides, A.A. (2011), "The acceptance and use of computer based assessment", Computers \& Education, Vol. 56, No. 4, pp. 1032-1044. 
Tibenderana, P., Ogao, P., Ikoja-Odongo, J. and Wokadala, J. (2010), "Measuring Levels of EndUsers' Acceptance and Use of Hybrid Library Services", International Journal of Education and Development using Information and Communication Technology, Vol. 6, No. 2, pp. 1-22.

Trimmer, K., Beachboard, J., Wiggins, C. and Woodhouse, W. (2008), "Electronic Medical Records Use - An Examination of Resident Physician Intentions", Proceedings of the $41^{\text {st }}$ Hawaii International Conference on System Sciences, pp. 1-10.

Tsai, Y.H., Lin, C.P., Chiu, C.K. and Joe, S.W. (2009), "Understanding learning behavior using location and prior performance as moderators", Social Science Journal, Vol. 46, No. 4, pp. 787-799.

Udeh, E.P. (2008), "Exploring user acceptance of free wireless fidelity public hot spots: An empirical study", An Interdisciplinary Journal on Humans in ICT Environments, Vol. 4, No. 2, pp. 144-168.

Uzoka, F.M.E. (2008), "Organizational Influences on E-commerce adoption in a developing country context using UTAUT”, International Journal of Business Information Systems, Vol. 3, No, 3, pp. 300-316.

van Biljon, J. and Kotze, P. (2008), "Cultural Factors in a Mobile Phone Adoption and Usage Model”, Journal of Universal Computer Science, Vol. 14, No. 16, pp. 2650-2679.

van Biljon, J. and Renaud, K. (2008), "A Qualitative Study of the Applicability of Technology Acceptance Models to Senior Mobile Phone Users", in I.Y. Song (Ed.), Advances in Conceptual Modeling - Challenges and Opportunities. Berlin: Springer-Verlag Berlin Vol. 5232, pp. 228-237.

van Dijk, J., Peters, O. and Ebbers, W. (2008), "Explaining the acceptance and use of government Internet services: A multivariate analysis of 2006 survey data in the Netherlands", Government Information Quarterly, Vol. 25, No. 3, pp. 379-399.

van Raaij, E.M. and Schepers, J.J.L. (2008), "The acceptance and use of a virtual learning environment in China", Computers \& Education, Vol. 50, No. 3, pp. 838-852.

van Schaik, P. (2009), "Unified Theory of Acceptance and Use for Websites Used by Students in Higher Education”, Journal of Educational Computing Research, Vol. 40, No. 2, pp. 229-257.

van Setten, M., Veenstra, M., Nijholt, A. and van Dijk, B. (2006), "Goal-based restructuring in recommender systems", Interacting with Computers, Vol. 18, pp. 432-456.

Venkatesh, V., Brown, S.A., Maruping, L.M. and Bala, H. (2008), "Predicting different conceptualizations of system use: The competing roles of behavioral intention, facilitating conditions, and behavioral expectation", MIS Quarterly, Vol. 32, No. 3, pp. 483-502.

Venkatesh, V., Morris, M.G., Davis, G.B. and Davis, F.D. (2003), "User acceptance of information technology: Toward a unified view”, MIS Quarterly, Vol. 27, No. 3, pp. 425-478.

Verhoeven, J.C., Heerwegh, D. and Wit, K.D. (2010), "Information and communication technologies in the life of university freshmen: An analysis of change", Computers \& Education, Vol. 55, No. 1, pp. 53-66.

Wang, H-Y., Chan, T-J., Huang, Y-F., Wang, N-C. and Chang, Y-S. (2010), "Research Hypotheses for Gender Activities in Mobile Internet", IWCMC'10, Caen, France.

Wang, Y.S. and Shih, Y.W. (2009), "Why do people use information kiosks? A validation of the Unified Theory of Acceptance and Use of Technology", Government Information Quarterly, Vol. 26, No. 1, pp. 158-165.

Wang, Y.S., Wu, M.C. and Wang, H.Y. (2009), "Investigating the determinants and age and gender differences in the acceptance of mobile learning", British Journal of Educational Technology Vl. Vol. 40, No. 1, pp. 92-118.

Weerakkody, V., Al-Shafi, S., Irani, Z. and Lee, H. (2009), "G-government adoption in Qatar: An investigation of the citizens' perspective", DIGIT Proceedings.

Weinstein, N.D., (1980), “Optimistic bias about personal risks”, Science 246, pp. 1232-1233.

Whitten, P., Holtz, B., Meyer, E., Nazione, S. (2009), "Telehospice: reasons for slow adoption in home hospice care", Journal of Telemedicine and Telecare, Vol. 15, No. 4, pp. 187-190. 
Williams, M.D., Dwivedi, Y.K., Lal, B. and Schwarz, A. (2009), "Contemporary Trends and Issues in IT Adoption and Diffusion Research", Journal of Information Technology, Vol. 24, No. 1, pp. 1-10.

Wills, M.J., El-Gayar, O.F. and Bennett, D. (2008), "Examining healthcare professionals' acceptance of electronic medical records using UTAUT", Issues in Information Systems, Vol. 9, No. 2, pp. 396401.

Wu, D., Hiltz, S.R. and Bieber, M. (2010), "Acceptance of Educational Technology: Field Studies of Asynchronous Participatory Examinations", Communications of the Association for Information Systems, Vol. 26, No. 1, pp. 451-476.

Wu, Y.L., Tao, Y.H. and Yang, P.C. (2007), "Using UTAUT to explore the behavior of 3G mobile communication users", In M. Helander, M. Xie, M. Jaio and K.C. Tan (Eds.): International Conference on Industrial Engineering and Engineering Management, Vol. 1, No. 4, pp. 199-203.

Wu, Y-L., Tao, W-H. and Yang, P-C. (2008), "The use of unified theory of acceptance and use of technology to confer the behavioural model of $3 \mathrm{G}$ mobile telecommunication users", Journal of Statistics \& Management Systems, Vol. 11, No. 5, pp. 919-949.

$\mathrm{Xu}, \mathrm{H}$. and Gupta, S. (2009), "The effects of privacy concerns and personal innovativeness on potential and experienced customers' adoption of location-based services", Electronic Markets, Vol. 19, No. 2-3, pp. 137-149.

Yang, D., Wang, Q., Li, M.S., Yang, Y., Ye, K. and Du, J. (2008), A Survey on Software Cost Estimation in the Chinese Software Industry. New York: Association of Computing Machinery.

Yang, K. (2010), "Determinants of US consumer mobile shopping services adoption: implications for designing mobile shopping services", Journal of Consumer Marketing, Vol. 27, No. 3, pp. 262-270.

Yao, Y.R. and Murphy, L. (2007), "Remote electronic voting systems: An exploration of voters' perceptions and intention to use", European Journal of Information Systems, Vol. 16, No. 2, pp. 106120.

YenYuen, Y. and Yeow, P.H.P. (2009), "User Acceptance of Internet Banking Service in Malaysia", In J. Cordeiro, S. Hammoudi and J. Filipe (Eds.): Web Information Systems and Technologies. Berlin: Springer-Verlag Berlin, Vol. 18, pp. 295-306.

Yeow, P.H.P. and Loo, W.H. (2009), "Acceptability of ATM and Transit Applications Embedded in Multipurpose Smart Identity Card: An Exploratory Study in Malaysia", International Journal of Electronic Government Research, Vol. 5, No. 2, pp. 37-56.

Yeow, P.H.P., Yuen, Y.Y., Tong, D.Y.K. and Lim, N. (2008), "User acceptance of Online Banking Service in Australia", Norristown: International Business Information Management Association.

Zhang, J., Huang, J. and Chen, J. (2010), "Empirical Research on User Acceptance of Mobile Searches", Tsinghua Science \& Technology, Vol. 15, No. 2, pp. 235-245.

Zhou, T. (2008), "Exploring Mobile User Acceptance Based on UTAUT and Contextual Offering", International Symposium on Electronic Commerce and Security, pp. 241-245.

Zhou, T., Lu, Y.B. and Wang, B. (2010), "Integrating TTF and UTAUT to explain mobile banking user adoption", Computers in Human Behavior, Vol. 26, No. 4, pp. 760-767. 
Appendix A.

Table 16

Methodological Details (Approach from Legris et al., 2003)

\begin{tabular}{|c|c|c|c|}
\hline Study & System/Software/Application Type & Sample Size & Model(s) Used \\
\hline AbuShanab and Pearson (2007) & Internet Banking & 523 non-Internet banking customers & \begin{tabular}{|l|} 
UTAUT \\
\end{tabular} \\
\hline Abu-Shanab and Pearson (2009) & Internet Banking & 878 bank customers & $\begin{array}{l}\text { UTAUT } \\
\end{array}$ \\
\hline AbuShanab et al. (2010) & Internet Banking & 523 non-Internet banking customers & UTAUT \\
\hline Aggelidis and Chatzoglou (2009) & Hospital Information System & 283 employees & \begin{tabular}{|l} 
UTAUT+TAM \\
\end{tabular} \\
\hline Alapetite et al. (2009) & Speech Recognition System & 39 physicians & \begin{tabular}{|l} 
UTAUT \\
\end{tabular} \\
\hline AlAwadhi and Morris (2011) & E-Government Services & 880 students & UTAUT \\
\hline Al-Gahtani et al. (2007) & Information Technology & 722 knowledge workers & UTAUT+Theory of Cultural Dimension \\
\hline Al-Harby et al. (2010) & Biometrics Authentication System & 306 under/post-graduate students & UTAUT \\
\hline Al-Rajhi et al. (2010) & Information System & Study not validated yet & UTAUT \\
\hline Al-Shafi and Weerakkody (2009) & E-Government Services & 216 citizens & $\begin{array}{l}\text { UTAUT } \\
\end{array}$ \\
\hline Al-Shafi and Weerakkody (2010) & E-Government Services & 1179 citizens & UTAUT \\
\hline Al-Sobhi et al. (2011) & E-Government Services & 750 citizens & UTAUT \\
\hline Al-Somali et al. (2009) & Online Banking & 202 bank customers & \begin{tabular}{|l} 
UTAUT+TAM2 \\
\end{tabular} \\
\hline Ambali (2009) & E-Filing System & 300 taxpayers & UTAUT+TAM \\
\hline Anderson et al. (2006) & Tablet PCs & 37 faculty members & UTAUT \\
\hline Aoun et al. (2010) & Accounting Information System & 192 accounting practitioners & UTAUT \\
\hline Avdic and Eklund (2010) & Reference Databases & 150 students & UTAUT \\
\hline Bandyopadhyay and Bandyopadhyay (2010) & Information Technology & 762,502 professionals & \begin{tabular}{|l} 
UTAUT \\
\end{tabular} \\
\hline Barati and Mohammadi (2009) & Mobile Banking & Exploratory Study-data to be collected & \begin{tabular}{|l} 
UTAUT+TAM \\
\end{tabular} \\
\hline Barnes and Vidgen (2009) & Corporate Intranet & 131 sales and marketing professionals & UTAUT+TAM \\
\hline BenMessaoud et al. (2011) & Robotic-Assisted Surgery & 21 surgeons & UTAUT \\
\hline Benslimane et al. (2004) & Web Systems for e-Procurement & 136 corporate buyers & \begin{tabular}{|l} 
UTAUT+TAM \\
\end{tabular} \\
\hline Biemans et al. (2005) & Medical Teleconferencing Application & 18 nurses & UTAUT \\
\hline Brown and Venkatesh (2005) & Information Technology & 746 households & UTAUT \\
\hline Brown et al. (2010) & Collaboration Technology & 349 SMS users & UTAUT \\
\hline Butler and Richardson (2008) & iBrainz Technology & 47 students & $\begin{array}{l}\text { UTAUT } \\
\end{array}$ \\
\hline Cabral et al. (2009) & Water Treatment Technology & No data value collected yet & UTAUT+TAM \\
\hline Carlsson et al. (2006) & Mobile Devices/Services & 157 subjects & UTAUT \\
\hline Carter and Schaupp (2009) & E-File & 260 students & $\begin{array}{l}\text { UTAUT } \\
\end{array}$ \\
\hline Chan et al. (2010) & E-Government Technology & 1179 citizens & UTAUT \\
\hline Chang et al. (2007) & Clinical Decision Support System & 140 physicians & UTAUT \\
\hline Chen et al. (2008) & Weblog System & 153 students & UTAUT \\
\hline Cheng et al. (2008a) & Internet Banking & 413 professionals & UTAUT \\
\hline
\end{tabular}


Cheng et al. (2008b)

Cheng et al. (2008c)

Chisolm et al. (2010)

Chiu and Eysenbach (2010)

Chiu and Wang (2008)

Chiu et al. (2010)

Cody-Allen and Kishore (2006)

Cornacchia et al. (2008)

Coss (2009)

Curtis and Payne (2008)

Curtis et al. (2010)

Dada (2006)

Dadayan and Ferro (2005)

Dasgupta and Gupta (2010)

de Silva and Ratnadiwakara (2009)

Debuse et al. (2008)

Diaz and Loraas (2010)

Dulle and Minishi-Majanja (2011)

Duyck et al. (2008)

Duyck et al. (2010)

Fitterer et al. (2010)

Foon and Fah (2011)

Gunther et al. (2009)

Gupta et al. (2008)

Hailemariam et al. (2010)

$\mathrm{He}$ and $\mathrm{Lu}(2007 \mathrm{a})$

$\mathrm{He}$ and $\mathrm{Lu}(2007 \mathrm{~b})$

$\mathrm{He}$ and Wei (2009)

Heerink et al. (2009)

Heikkila and Smale (2010)

Hennington et al. (2009)

Ho and Chou (2009)

Huang and Wang (2009)

Huang et al. (2010)

Hung et al. (2007)

Huser et al. (2010)

Hutchison and Bekkering (2009)
Internet Banking

Internet Banking

Electronic Medical Record

E-Health Services

Web-Based Learning

Online Auctions

E-Quality

ICT

Medical Support System

Computer-Assisted Audit Techniques

Social Media

E-Readiness

Technology

Internet Technology

Mobile Technology

Automated Feedback System

Existing Technology

Open Access

PACS System

PACS System

Health Information System

Internet Technology

Micro Blogging

ICT

Telemedicine

Mobile Advertising

Mobile Business

Knowledge Management System

Interface Robot

Electronic HRM System

Electronic Medical Record System

Mobile Podcasting

ERP System

Information Technology

E-Government Services

EHR Query System

Remote Desktop Application
313 professionals

313 professionals

71 clinicians

46 professionals

286 respondents

412 buyers

Data collection to be done in the future

40 employees

Data collection to be done in the future

139 professionals

409 professionals

328 people from Tanzania+78 from SA

Data collection will be done latter

102 government employees

9540 telephone users

8 academic staff

69 students

544 teachers

56 professionals

203, 159, 362 Physicians-Radiologists

79 professionals

200 professionals

25 Twitter Users

102 employees

Physicians and health workers

243 mobile consumers

74 journal articles

161 professionals

42 elderly citizens

18 HR managers

23 Nurses and 4 Nurse Managers

246 citizens

236 professionals

Healthcare professionals from 10 firms

244 citizens

18 human subjects

25 students
UTAUT

UTAUT+DeLone \& McLean ISS Model

UTAUT

UTAUT+Andersen's Behavioral Model

UTAUT

UTAUT

UTAUT+DeLone \& McLean ISS Model

UTAUT

UTAUT

UTAUT

UTAUT

UTAUT

UTAUT+TAM

UTAUT

UTAUT+TAM

UTAUT

UTAUT

UTAUT

UTAUT

UTAUT

UTAUT

UTAUT

UTAUT

UTAUT

UTAUT

UTAUT+IDT+TTF

UTAUT+TAM+TPB+TTF

UTAUT

UTAUT

UTAUT

UTAUT

UTAUT

UTAUT+TAM

UTAUT

UTAUT

UTAUT+TAM

UTAUT 
Im et al. (2008)

Jalaldeen et al. (2009)

Jayasingh and Eze (2009)

Johari et al. (2010)

Johnston and Warkentin (2010)

Jong and Wang (2009)

Keller et al. (2007)

Kijsanayotin et al. (2009)

Koh et al. (2010)

Koivumaki et al. (2008)

Kourouthanassis et al. (2010)

Laumer et al. (2010)

Lee and Lin (2008)

Lee et al. (2007)

Lee et al. (2010a)

Lee et al. (2010b)

$\mathrm{Li} \mathrm{(2010)}$

Li and Kishore (2006)

Lin et al. (2004)

Liu et al. (2008)

Loo et al. (2009)

Louho et al. (2006)

Lubrin et al. (2006)

Luo et al. (2010)

Mahzan and Lymer (2008)

Maldonado et al. (2009)

Maldonado et al. (2011)

Marchewka et al. (2007)

Mayer et al. (2011)

McLeod et al. (2009a)

McLeod et al. (2009b)

Neufeld et al. (2007)

Niehaves and Plattfaut (2010)

Nistor et al. (2010)

Nov and Ye (2009)

Or et al. (2011)

Oshlyansky et al. (2007)
Technologies for Communication Knowledge Management Process M-Coupon System

Information Kiosk

Security Information System

Web-based Learning System

E-Learning System

Health Information Technology

Software Technologies

Mobile Services

Mobile Internet Application

Information Technology

Podcasting

Forecasting Support System

Activity Based Management System

DEMATEL

Virtual Knowledge Sharing

Online Community Weblog System

Instant Messaging

Internet banking

Smartcard Application

Hybrid Media Application

Motes

Mobile Banking

CAATTs

Educational Portal

Educational Portal

Course Management Software

Smart Products

Tax Software System

Tax Preparation Software

Information Technology

Internet

E-Learning System

Digital Library

Web-Based Technology

Validating UTAUT tool
161 subjects

Conceptual model - to be used latter

781 mobile consumers

Data collection to be done in the future

275 subjects

606 students

67 students

1187 community health centres

333 employees

243 mobile consumers

139 subscribers

323 students

190 students

54 students

112 professionals

10 professionals

41 employees

265 students

300 students

413 professionals

200 MyKad holders

19 test users

103 anonymous participants

122 students

46 members of IIA-UK

150 students

150 students

132 students

166 citizens

74 professionals and 56 novices

215 students

209 professionals

192 elderly citizens

732 students

271 students

101 patients

1489 students from nine countries
UTAUT+TAM

UTAUT

UTAUT+TAM2

UTAUT

UTAUT

UTAUT

UTAUT+IDT

UTAUT

UTAUT+DeLone \& McLean ISS Mode

UTAUT

UTAUT+TPB+DOI

UTAUT

UTAUT+TAM

UTAUT

UTAUT

UTAUT+TAM2

UTAUT

UTAUT

UTAUT

UTAUT+DeLone \& McLean ISS Model +TM

UTAUT

UTAUT

UTAUT

UTAUT

UTAUT

UTAUT

UTAUT

UTAUT

UTAUT

UTAUT

UTAUT

UTAUT

UTAUT

UTAUT

UTAUT

UTAUT+TAM+TPB

UTAUT+TAM 
Pahlke and Beck (2009)

Pahnila et al. (2011)

Pai and Tu (2011)

Pappas and Volk (2007)

Park et al. (2007)

Pavon and Brown (2010)

Payne (2008)

Pynoo et al. (2008)

Pynoo et al. (2011)

Qingfei et al. (2008)

Randeree (2009)

Reunis and Santema (2005)

Richardson et al. (2009)

Sahu and Gupta (2007)

Sambasivan et al. (2010)

Samoutis et al. (2008)

Sapio et al. (2010)

Schaper and Pervan (2004)

Schaper and Pervan (2006)

Schaupp et al. (2010)

Seymour et al. (2007)

Shamsuddin (2009)

Sharma and Citurs (2004)

Shi (2009)

Shin (2009)

Shin (2010)

Song and Han (2009)

Suhendra et al. (2009)

Suki and Ramayah (2010)

Sumak et al. (2010)

Taksa and Flomenbaum (2009)

Tan and $\mathrm{Wu}(2010)$

Tavares and Amarel (2010)

Teo (2011)

Terzis and Economides (2011)

Tibenderana et al. (2010)

Trimmer et al. (2008)
Enterprise Mashup System

Auction Site

CRM Systems

Audience Counts \& Reporting System

Mobile Technology

World Wide Web

Web 2.0

PACS System

Digital Learning Environment

Mobile Commerce

Personal Health Record System

E-Ordering Application

Problem Solving Models

E-Government

Electronic Procurement System

Quality Improvement Intervention

Digital Television ICT

Technologies

E-File

Enterprise Resource Planning Systems

Computer Graphics Technology

Information Technology

Smart Phone Application Software

Mobile Wallet

MVNO Services

Mobile System

Information Technology

E-Government Services

Virtual Learning Environment

Cross-Cultural Information Retrieval

Mobile Commerce

Peer-to-Peer Academic Networks

Intention to Use Technology

Computer Based Assessment Model

Hybrid Library Services

Electronic Medical Record Systems
Constructs to be operationalized latter 180 students

271 professionals

27 independent education organizations

221 citizens

228 job seekers

(3)

600,180 physicians

64, 41, 55 teachers

Data collection and analysis to be done

128 students

25 professionals

33 students

163 users of Indian central excise

358 users from various ministries

18 patients

181 citizens

6500 professionals

2870 professionals

260 taxpayers

59 students

46 students

Proposed model would be tested latter

653 professionals

296 professionals

296 members of community

570 consumers

150 SME operators

200 respondents

235 student

20 Websites

300 students

10 interviews from users and non-user

592 teachers

173 students

445 staff and students

Data collection in process
UTAUT

UTAUT

UTAUT+TTF

UTAUT

UTAUT

UTAUT+TAM

UTAUT

UTAUT

UTAUT

UTAUT

UTAUT

UTAUT+TAM

UTAUT+DeLone \& McLean ISS Model

UTAUT+TRA+TPB

UTAUT

UTAUT

UTAUT+TAM

UTAUT

UTAUT

UTAUT

UTAUT

UTAUT

UTAUT+TAM

UTAUT+IDT+TAM

UTAUT

UTAUT

UTAUT+TAM

UTAUT

UTAUT

UTAUT+TAM

UTAUT

UTAUT+TAM+TPB

UTAUT+TAM+TPB

UTAUT

UTAUT 


\begin{tabular}{|c|c|c|c|}
\hline Tsai et al. (2009) & Learning Behaviour Formation & 759 students & UTAUT+SCT \\
\hline Udeh (2008) & Wi-Fi System & 129 respondents & UTAUT+TAM \\
\hline Uzoka (2008) & E-Commerce & 150 organizations & UTAUT \\
\hline van Biljon and Kotze (2008) & Mobile Phone & 59 students & UTAUT+TAM \\
\hline van Biljon and Renaud (2008) & Mobile Phone & 34 elderly citizens & UTAUT \\
\hline van Dijk et al. (2008) & Government Internet Services & 1225 respondents & UTAUT \\
\hline van Raaij and Schepers (2008) & Virtual Learning Environment & 45 students & UTAUT+TAM+TAM2 \\
\hline van Schaik (2009) & Websites & 118,121 students & UTAUT \\
\hline van Setten et al. (2006) & Recommender System & 1872 television viewers & UTAUT \\
\hline Venkatesh et al. (2008) & New System Use & 321 employees & UTAUT+TAM2 \\
\hline Verhoeven et al. (2010) & ICT & 714 students & UTAUT \\
\hline Wang and Shih (2009) & Information Kiosks & 244 respondents & UTAUT \\
\hline Wang et al. (2009) & Mobile Learning & 330 Respondents with IT experience & UTAUT \\
\hline Wang et al. (2010) & Mobile Internet & 343 respondents & UTAUT \\
\hline Weerakkody et al. (2009) & E-Government & 1179 citizens & UTAUT \\
\hline Whitten et al. (2009) & Telehospice & 25 employees & UTAUT \\
\hline Wills et al. (2008) & Electronic Medical Record & 52 professionals & UTAUT \\
\hline Wu et al. (2007) & 3G Mobile Communication & 394 professionals & UTAUT \\
\hline Wu et al. (2008) & 3G Mobile Telecommunication & 394 professionals & UTAUT \\
\hline Wu et al. (2010) & Educational Technology System & 240 students & UTAUT+TAM \\
\hline Xu and Gupta (2009) & Location-Based Services & 101 students & UTAUT \\
\hline Yang (2010) & Mobile Shopping Services & 400 mobile consumers & UTAUT \\
\hline Yang et al. (2008) & Software Cost Estimation & 116 organizations & UTAUT \\
\hline Yao and Murphy (2007) & Remote Electronic Voting Systems & $453,253,196$ voters & UTAUT+TAM \\
\hline YenYuen and Yeow (2009) & Internet Banking & 280 general users & UTAUT \\
\hline Yeow and Loo (2009) & ATM and Transit Application & 500 MyKad holders & UTAUT \\
\hline Yeow et al. (2008) & Online Banking Service & 190 respondents & UTAUT \\
\hline Zhang et al. (2010) & Mobile Search Service & 195 students & UTAUT+TTF \\
\hline Zhou (2008) & Mobile Commerce & 250 mobile commerce users & UTAUT \\
\hline Zhou et al. (2010) & Mobile Banking & 250 students and professionals & UTAUT+TTF \\
\hline
\end{tabular}

[Legend: CAATTs: Computer Assisted Audit Tools and Techniques (CAATTs), DeLone \& McLean ISS Model: DeLone and McLean IS Success Model, DEMATEL: Decision Making Trial and Evaluation Laboratory System, ICT: Information and Communication Technology, IDT: Innovation Diffusion Theory, TM: Trust Model, TTF: Task Technology Fit, Italic font: under sample size indicates that sample data have not been collected, analysed, or validated] 